Document downloaded from:

http://hdl.handle.net/10251/64857

This paper must be cited as:

Vico Bondía, F.; Ferrando Bataller, M.; Greengard, L.; Gimbutas, Z. (2016). The decoupled potential integral equation for time harmonic electromagnetic scattering. Communications on Pure and Applied Mathematics. 69(4):771-812. doi:10.1002/cpa.21585.

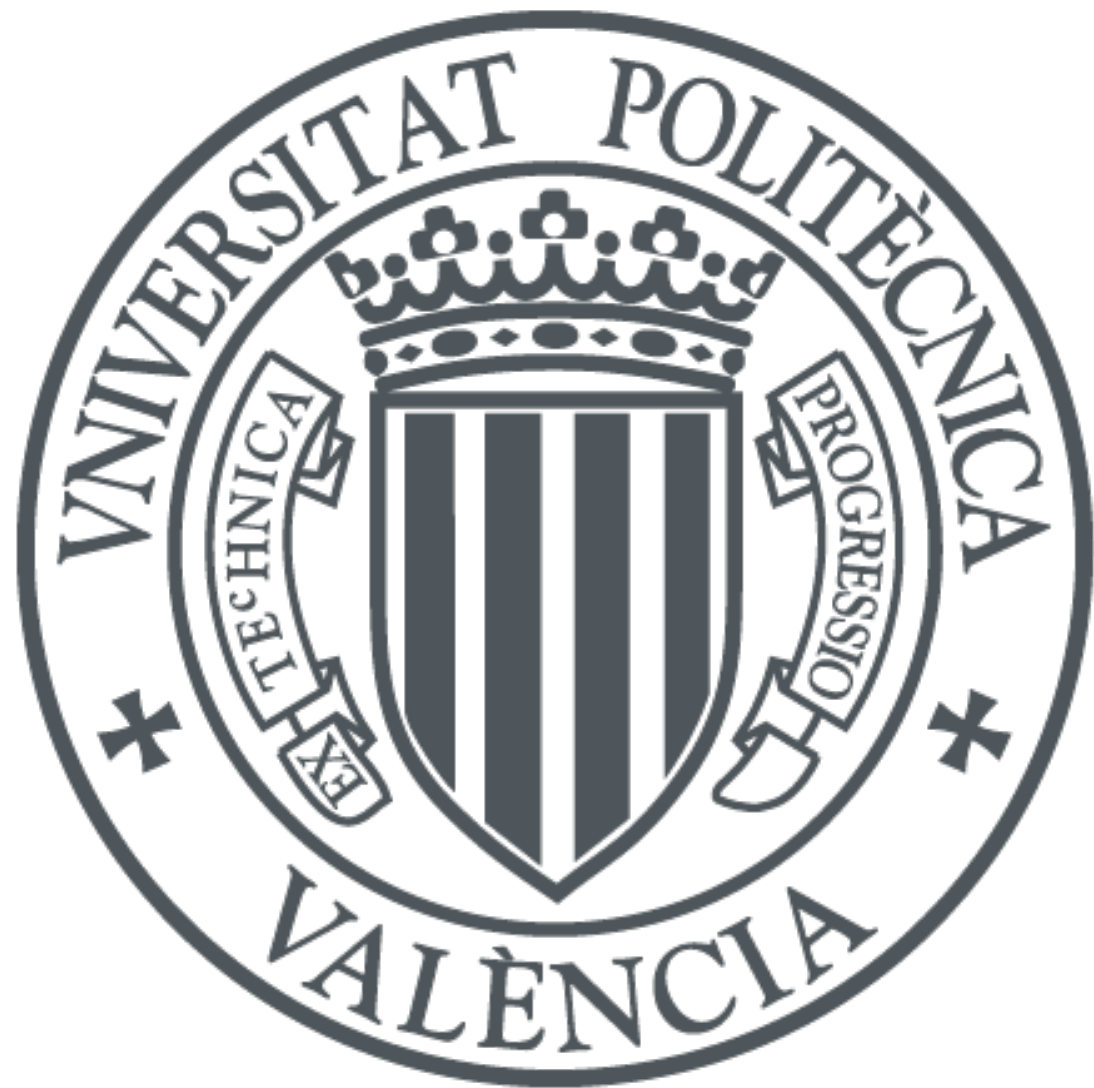

The final publication is available at

http://dx.doi.org/10.1002/cpa.21585

Copyright Wiley: 12 months

Additional Information

This is the peer reviewed version of the following article: "Vico, F., Ferrando, M., Greengard, L. and Gimbutas, Z. (2016), The Decoupled Potential Integral Equation for Time-Harmonic Electromagnetic Scattering. Commun. Pur. Appl. Math., 69: 771-812", which has been published in final form at http://dx.doi.org/10.1002/cpa.21585. This article may be used for non-commercial purposes in accordance with Wiley Terms and Conditions for SelfArchiving. 


\title{
The Decoupled Potential Integral Equation for Time-Harmonic Electromagnetic Scattering
}

\author{
FELIPE VICO \\ Instituto de Telecomunicaciones y Aplicaciones Multimedia (ITEAM), \\ Universidad Politècnica de València \\ MIGUEL FERRANDO \\ Instituto de Telecomunicaciones y Aplicaciones Multimedia (ITEAM), \\ Universidad Politècnica de València \\ LESLIE GREENGARD \\ Simons Center for Data Analysis, Simons Foundation \\ Courant Institute \\ AND \\ ZYDRUNAS GIMBUTAS \\ Information Technology Laboratory, National Institute of Standards and Technology
}

\begin{abstract}
We present a new formulation for the problem of electromagnetic scattering from perfect electric conductors. While our representation for the electric and magnetic fields is based on the standard vector and scalar potentials $\boldsymbol{A}, \phi$ in the Lorenz gauge, we establish boundary conditions on the potentials themselves rather than on the field quantities. This permits the development of a wellconditioned second-kind Fredholm integral equation that has no spurious resonances, avoids low-frequency breakdown, and is insensitive to the genus of the scatterer. The equations for the vector and scalar potentials are decoupled. That is, the unknown scalar potential defining the scattered field, $\phi^{\text {scat }}$, is determined entirely by the incident scalar potential $\phi^{\text {inc }}$. Likewise, the unknown vector potential defining the scattered field, $\boldsymbol{A}^{\text {scat }}$, is determined entirely by the incident vector potential $\boldsymbol{A}^{\text {inc }}$. This decoupled formulation is valid not only in the static limit but for arbitrary $\omega \geq 0$. (C) 2015 Wiley Periodicals, Inc.
\end{abstract}

\section{Introduction}

In this paper, we consider the problem of exterior scattering of time-harmonic electromagnetic waves by perfect electric conductors. For a fixed frequency $\omega$, we assume that the electric and magnetic fields take the form

$$
\mathcal{E}(\boldsymbol{x}, t)=\mathfrak{R}\left\{\boldsymbol{E}(\boldsymbol{x}) e^{-i \omega t}\right\}, \quad \mathcal{H}(\boldsymbol{x}, t)=\mathfrak{R}\left\{\boldsymbol{H}(\boldsymbol{x}) e^{-i \omega t}\right\},
$$

so that Maxwell's equations are

$$
\nabla \times \boldsymbol{E}(\boldsymbol{x})=i \omega \mu \boldsymbol{H}(\boldsymbol{x}), \quad \nabla \times \boldsymbol{H}(\boldsymbol{x})=-i \omega \epsilon \boldsymbol{E}(\boldsymbol{x}) .
$$

Communications on Pure and Applied Mathematics, 0001-0042 (PREPRINT)

(C) 2015 Wiley Periodicals, Inc. 
Following standard practice, we write the total electric and magnetic fields as a sum of the (known) incident and (unknown) scattered fields:

$$
\boldsymbol{E}=\boldsymbol{E}^{\text {inc }}+\boldsymbol{E}^{\text {scat }}, \quad \boldsymbol{H}=\boldsymbol{H}^{\text {inc }}+\boldsymbol{H}^{\text {scat }} .
$$

The scattered field in the exterior must satisfy the Sommerfeld-Silver-Müller radiation condition:

$$
\boldsymbol{H}^{\mathrm{scat}}(\boldsymbol{x}) \times \frac{\boldsymbol{x}}{|\boldsymbol{x}|}-\sqrt{\frac{\mu}{\epsilon}} \boldsymbol{E}^{\mathrm{scat}}(\boldsymbol{x})=o\left(\frac{1}{|\boldsymbol{x}|}\right), \quad|\boldsymbol{x}| \rightarrow \infty .
$$

uniformly for all directions $\frac{\boldsymbol{x}}{|\boldsymbol{x}|}$.

It is well-known that when the scatterer, denoted by $D$, is a perfect conductor, the conditions to be enforced on its boundary are [17,26]

$$
\begin{gathered}
\boldsymbol{n} \times \boldsymbol{E}(\boldsymbol{x})=\left.\boldsymbol{0}\right|_{\partial D} \Rightarrow \boldsymbol{n} \times \boldsymbol{E}^{\mathrm{scat}}(\boldsymbol{x})=-\boldsymbol{n} \times\left.\boldsymbol{E}^{\mathrm{inc}}(\boldsymbol{x})\right|_{\partial D}, \\
\boldsymbol{n} \cdot \boldsymbol{H}(\boldsymbol{x})=\left.0\right|_{\partial D} \Rightarrow \boldsymbol{n} \cdot \boldsymbol{H}^{\mathrm{scat}}(\boldsymbol{x})=-\left.\boldsymbol{n} \cdot \boldsymbol{H}^{\mathrm{inc}}(\boldsymbol{x})\right|_{\partial D},
\end{gathered}
$$

where $\boldsymbol{n}$ is the outward unit normal to the boundary $\partial D$ of the scattered. It is also well-known that

$$
\begin{gathered}
\boldsymbol{n} \cdot \boldsymbol{E}(\boldsymbol{x})=\left.\frac{\rho}{\epsilon}\right|_{\partial D}, \\
\boldsymbol{n} \times \boldsymbol{H}(\boldsymbol{x})=\left.\boldsymbol{J}\right|_{\partial D},
\end{gathered}
$$

where $\boldsymbol{J}$ and $\rho$ are the induced current density and charge on the surface $\partial D$. In order to satisfy the Maxwell equations, $\boldsymbol{J}$ and $\rho$ must satisfy the continuity condition $\nabla_{s} \cdot \boldsymbol{J}=i \omega \rho$, where $\nabla_{s} \cdot \boldsymbol{J}$ denotes the surface divergence of the tangential current density. It is also well-known that the exterior problem for $\boldsymbol{E}^{\text {scat }}$ has a unique solution for $\omega>0$ when boundary conditions are prescribed on its tangential components (see, for example, [11,25]):

$$
\boldsymbol{n} \times \boldsymbol{E}^{\mathrm{scat}}(\boldsymbol{x})=\left.\mathbf{f}(\mathbf{x})\right|_{\partial D}
$$

for an arbitrary tangential vector field $\mathbf{f}$. On a perfect conductor, $\mathbf{f}(\mathbf{x})=-\boldsymbol{n} \times$ $\boldsymbol{E}^{\text {inc }}(\boldsymbol{x})$ to enforce $(1.5)$.

\subsection{The Vector and Scalar Potential}

Scattered electromagnetic fields are typically represented in terms of the induced surface current $\boldsymbol{J}$ and charge $\rho$ using the vector and scalar potentials in the Lorenz gauge:

$$
\begin{aligned}
\boldsymbol{E}^{\mathrm{scat}} & =i \omega \boldsymbol{A}^{\mathrm{scat}}-\nabla \phi^{\mathrm{scat}}, \\
\boldsymbol{H}^{\mathrm{scat}} & =\frac{1}{\mu} \nabla \times \boldsymbol{A}^{\mathrm{scat}}
\end{aligned}
$$


where

$$
\begin{gathered}
\boldsymbol{A}^{\mathrm{scat}}[\boldsymbol{J}](\boldsymbol{x})=\mu S_{k}[\boldsymbol{J}](\boldsymbol{x}) \equiv \mu \int_{\partial D} g_{k}(\boldsymbol{x}-\boldsymbol{y}) \boldsymbol{J}(\boldsymbol{y}) d A_{\boldsymbol{y}}, \\
\phi^{\text {scat }}[\rho](\boldsymbol{x})=\frac{1}{\epsilon} S_{k}[\rho](\boldsymbol{x}) \equiv \frac{1}{\epsilon} \int_{\partial D} g_{k}(\boldsymbol{x}-\boldsymbol{y}) \rho(\boldsymbol{y}) d A_{\boldsymbol{y}},
\end{gathered}
$$

with

$$
g_{k}(\boldsymbol{x})=\frac{e^{i k|\boldsymbol{x}|}}{4 \pi|\boldsymbol{x}|}
$$

and $k=\omega \sqrt{\epsilon \mu}$. We chose the sign of $k$ such that $\operatorname{Im}(k) \geq 0$. Here, $\operatorname{Im}(k)$ denotes the imaginary part of $k$. The Lorenz gauge is defined by the relation

$$
\nabla \cdot \boldsymbol{A}^{\mathrm{scat}}=i \omega \mu \epsilon \phi^{\mathrm{scat}} \text {. }
$$

We will often refer to $\phi^{\text {scat }}$ and $\boldsymbol{A}^{\text {scat }}$ as the scalar and vector Helmholtz potentials since $\phi^{\text {scat }}$ and $\boldsymbol{A}^{\text {scat }}$ satisfy the Helmholtz equations with wavenumber $k$ :

$$
\Delta \phi^{\text {scat }}+k^{2} \phi^{\text {scat }}=0, \quad \Delta \boldsymbol{A}^{\text {scat }}+k^{2} \boldsymbol{A}^{\text {scat }}=\mathbf{0} .
$$

Using the representation (1.10) for the electric field and imposing the boundary condition (1.5) results in the electric field integral equation (EFIE) [9, 18, 23, 24]:

$$
i \omega \boldsymbol{n} \times \boldsymbol{A}^{\text {scat }}[\boldsymbol{J}](\boldsymbol{x})-\boldsymbol{n} \times \nabla \phi^{\text {scat }}\left[\frac{\nabla_{s} \cdot \boldsymbol{J}}{i \omega}\right](\boldsymbol{x})=-\boldsymbol{n} \times \boldsymbol{E}^{\mathrm{inc}}(\boldsymbol{x}), \quad \boldsymbol{x} \in \partial D .
$$

The representation (1.11) for the magnetic field and the boundary condition 1.8 results in the magnetic field integral equation (MFIE):

$$
\frac{1}{2} \boldsymbol{J}(\boldsymbol{x})-K[\boldsymbol{J}](\boldsymbol{x})=\boldsymbol{n}(\boldsymbol{x}) \times \boldsymbol{H}^{\mathrm{inc}}(\boldsymbol{x}), \quad \boldsymbol{x} \in \partial D,
$$

where

$$
K[\boldsymbol{J}](\boldsymbol{x})=\int_{\partial D} \boldsymbol{n}(\boldsymbol{x}) \times \nabla \times g_{k}(\boldsymbol{x}-\boldsymbol{y}) \boldsymbol{J}(\boldsymbol{y}) d A_{\boldsymbol{y}} .
$$

There is an enormous literature on the properties of these integral equations, which we will not review here, except to note that the EFIE is poorly scaled; one term in the representation of $\boldsymbol{E}$ is of order $O(\omega)$ and one term is of the order $O\left(\omega^{-1}\right)$. This makes it difficult to compute both the solenoidal and irrotational components of the current $\boldsymbol{J}$ and causes ill-conditioning in the integral equation at low frequencies, a phenomenon generally referred to as "low-frequency breakdown" [36, 37]. Both the EFIE and the MFIE are also subject to spurious resonances at a countable set of frequencies $\omega_{j}$ going to infinity. Below the first such resonance, the MFIE is a well-conditioned second-kind Fredholm integral equation. While low-frequency breakdown is obvious in the EFIE, it is not entirely avoided by switching to the MFIE [36]. The problem is that the current $\boldsymbol{J}$ is not sufficient for computing accurately the electric field. 
Note, for example, that

$$
\boldsymbol{n} \cdot \boldsymbol{E}=\rho=\frac{\nabla_{s} \cdot \boldsymbol{J}}{i \omega \epsilon} .
$$

As $\omega \rightarrow 0$, what in numerical analysis is called catastrophic cancellation causes a progressive loss of digits [19,20]. Catastrophic cancellation comes not just from the ill-conditioning associated with the evaluation of a derivative. The current $\boldsymbol{J}$ is an $O(1)$ quantity, while $\nabla_{s} \cdot \boldsymbol{J}$ is $O(\omega)$, amplifying the loss of digits.

A variety of remedies to solve this problem have been suggested. In the widest use are methods based on specialized basis functions for the discretization of the current $\boldsymbol{J}$ itself. Loop-tree and loop-star basis functions, for example, can be used to rescale the solenoidal and the irrotational parts of the current [22, 29, 33, 34, 37]. A second class of methods is based on using both current and charge as separate unknowns. This avoids terms of the order $O\left(\omega^{-1}\right)$ (see [6, 27, 28, 30, 35]). Unfortunately, all of these approaches encounter a second difficulty in multiply connected domains-a phenomenon that we refer to as "topological low-frequency breakdown" [10,13]. At zero frequency, the MFIE, Calderon-preconditioned EFIE, and charge-current based integral equations are all rank-deficient, with a nullspace of dimension related to the topology of the surface $\partial D: g$ for the MFIE, $2 g$ for the Calderon-preconditioned EFIE, and $g+N$ for the charge-current based integral equations [10,13,32], where $g$ is the genus of the surface $\partial D$ and $N$ is the number of connected components. This inevitably leads to ill-conditioning in the lowfrequency regime. This problem was carefully analyzed in the paper [13], and the nullspace characterized in terms of harmonic vector fields [10, 13, 32].

DEFinition 1.1. Assuming $D$ is topologically equivalent to a sphere with $g$ handles, one can choose $g$ surfaces $S_{j}$ in $\mathbb{R}^{3} \backslash D$ so that $\mathbb{R}^{3} \backslash\left(D \bigcup_{j=1}^{g} S_{j}\right)$ is simply connected. The boundaries of these surfaces are loops on $\partial D$ called $B$-cycles. They go around the "holes" and form a basis for the first homology group of the domain $D$.

In [14], it was shown that the addition of a consistency condition of the form

$$
\int_{S_{j}} \boldsymbol{H}^{\text {scat }} \cdot \boldsymbol{n} d a=-\int_{S_{j}} \boldsymbol{H}^{\mathrm{inc}} \cdot \boldsymbol{n} d a
$$

Or

$$
\int_{B_{j}} \boldsymbol{A}^{\mathrm{scat}} \cdot \boldsymbol{d} s=-\int_{B_{j}} \boldsymbol{A}^{\mathrm{inc}} \cdot \boldsymbol{d} s,
$$

where the line integrals represent the circulation of the vector potential, enforces uniqueness on the solution of the MFIE, assuming $\omega$ is not at a spurious resonance. This requires knowledge of, or computation of, the genus, geometry tools that identify $B$-cycles, and linear algebraic methods that are capable of efficiently solving integral equations subject to constraints. 


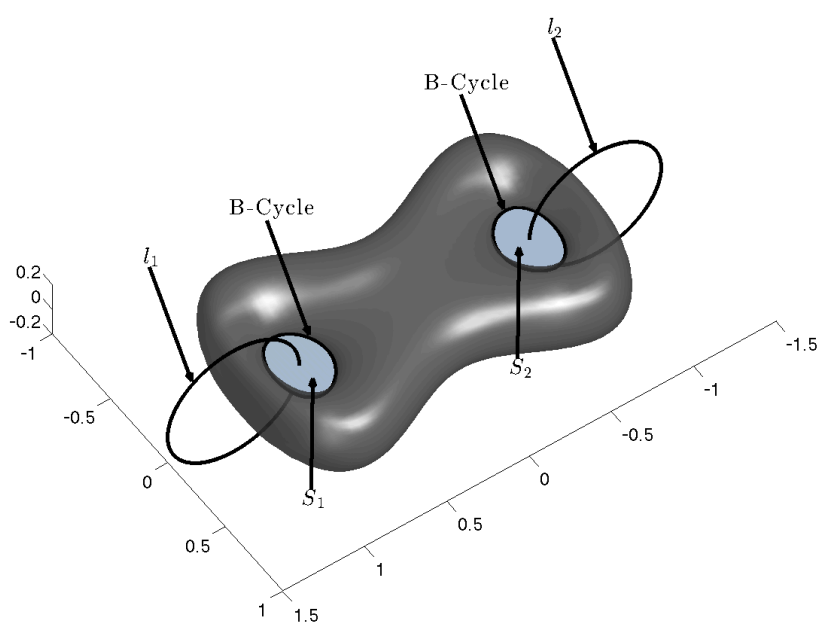

FIGURE 1.1. Double torus, number of connected components $N=1$, genus $g=2$, two B-cycles. $l_{1}$ and $l_{2}$ are the integration domain of integrals in 2.10). Surfaces $S_{1}$ and $S_{2}$ are covering each hole of the surface.

Remark 1.2. These topological issues can also be addressed through an analysis of the Hodge decomposition of the source current on the surface of the scatterer itself by using the generalized Debye source representation of [15]. Additional conditions are used (similar to 1.19 ) to ensure that the problem is well-posed.

Remark 1.3. Very recently a method was introduced that overcomes the topological low-frequency breakdown inherent in the EFIE by a clever projection of the discretized problem using Rao-Wilton-Glisson (RWG) basis functions into a suitable subspace [3].

In short, the various integral equations presently available pose significant difficulties in the low-frequency regime.

\subsection{A Decoupled Formulation}

In this paper we introduce a new formulation for electromagnetic scattering from perfect conductors. Rather than imposing boundary conditions on the field quantities $(\boldsymbol{E}, \boldsymbol{H})$, we derive conditions on the potentials themselves. Moreover, we show that the integral equations for $\boldsymbol{A}^{\text {scat }}$ and $\phi^{\text {scat }}$ can be decoupled, lead to wellconditioned linear systems, and are insensitive to the genus of the scatterer. More precisely, we seek to impose the boundary conditions

$$
\begin{gathered}
\boldsymbol{n} \times \boldsymbol{A}^{\mathrm{scat}}(\boldsymbol{x})=-\boldsymbol{n} \times\left.\boldsymbol{A}^{\mathrm{inc}}(\boldsymbol{x})\right|_{\partial D}, \\
\boldsymbol{n} \times \nabla \phi^{\text {scat }}(\boldsymbol{x})=-\boldsymbol{n} \times\left.\nabla \phi^{\mathrm{inc}}(\boldsymbol{x})\right|_{\partial D} .
\end{gathered}
$$

At first glance, there is an obvious difficulty with such an approach: the vector and scalar potentials are not unique, a fact generally referred to as gauge freedom. 
Even in the Lorenz gauge above, the representation is known not to be unique. That is, the condition 1.13 does not completely determine the potentials $A^{\text {scat }}, \phi^{\text {scat }}$.

To see this, consider the vector potentials $\boldsymbol{A}^{\prime \text { scat }}, \phi^{\prime \text { scat }}$ defined by

$$
\begin{aligned}
\boldsymbol{A}^{\prime \text { scat }}[\boldsymbol{J}](\boldsymbol{x}) & =\boldsymbol{A}^{\text {scat }}[\boldsymbol{J}](\boldsymbol{x})+\nabla S_{k}[\sigma](\boldsymbol{x}), \\
\phi^{\prime s c a t}[\rho](\boldsymbol{x}) & =\phi^{\text {scat }}[\rho](\boldsymbol{x})+i \omega S_{k}[\sigma](\boldsymbol{x}) .
\end{aligned}
$$

Here, $\sigma$ is an arbitrary source on the surface $\partial D$. It is straightforward to check that the fields $\boldsymbol{E}^{\text {scat }}, \boldsymbol{H}^{\text {scat }}$ induced by $\boldsymbol{A}^{\prime \text { scat }}, \phi^{\prime \text { scat }}$ are the same as those induced by $\boldsymbol{A}^{\text {scat }}, \phi^{\text {scat }}$, all the while satisfying the Lorenz gauge condition.

We will make use of this additional gauge freedom to establish a well-posed boundary value problem and a stable, well-conditioned integral equation. In Section 2, we consider the low-frequency limit of the exterior scattering from perfect conductors, both for the sake of review and to motivate our formulation. In Section 3. we discuss the relevant existence, uniqueness and stability results for what we refer to as the decoupled potential integral equation (DPIE). Finally, we discuss the stable representation of the incoming field in terms of scalar and vector potentials and the high-frequency behavior of the new formulation.

\section{Preliminaries}

In this section, we consider the low-frequency limit of the Maxwell equations, where the electric and magnetic fields are decoupled. We will refer to the electrostatic and magnetostatic fields by $\boldsymbol{E}_{0}$ and $\boldsymbol{H}_{0}$, respectively.

\subsection{Electrostatics}

The electrostatic field satisfies the equations

$$
\nabla \times \boldsymbol{E}_{0}(\boldsymbol{x})=0, \quad \nabla \cdot \boldsymbol{E}_{0}(\boldsymbol{x})=0, \quad \boldsymbol{x} \in \mathbb{R}^{3} / D,
$$

which we decompose (as above) into incoming and scattered fields. The scattered field must satisfy the radiation condition

$$
E_{0}^{\text {scat }}(\boldsymbol{x})=o(1), \quad|\boldsymbol{x}| \rightarrow \infty .
$$

The boundary condition for the electrostatic field is the same as that for any nonzero frequency,

$$
\boldsymbol{n} \times \boldsymbol{E}_{0}(\boldsymbol{x})=0, \quad \boldsymbol{x} \in \partial D,
$$

but the solution is no longer unique.

The nullspace, that is functions satisfying $(2.1)$ and $(2.3)$ and the radiation condition (2.2), is of dimension $N$, where $N$ is the number of connected components of the scatterers $\partial D$. They are known as harmonic Dirichlet fields [10] with a basis denoted by $\left\{\mathbf{Y}_{j}\right\}_{j=1}^{N}$. It is straightforward to see that there are at least $N$ such solutions, since they correspond to the well-studied problem of capacitance. To see this, let us denote by $\partial D_{j}$ the $j^{\text {th }}$ connected component of $\partial D$. Taking the 
static limit of (1.10), the scattered electrostatic field is described as the gradient of a scalar harmonic function:

$$
\boldsymbol{E}_{0}^{\mathrm{scat}}=-\nabla \phi_{0}^{\mathrm{scat}} \text {. }
$$

Imposing the boundary condition 2.3 and assuming the incoming field is represented in terms of an incoming potential $\phi^{\text {inc }}$, we have

$$
\Delta \phi_{0}^{\text {scat }}=0, \quad \boldsymbol{n} \times \nabla \phi_{0}^{\text {scat }}=-\boldsymbol{n} \times\left.\nabla \phi_{0}^{\text {inc }}\right|_{\partial D} .
$$

It is clear that the preceding boundary condition is satisfied by any scattered potential that satisfies the Dirichlet condition

$$
\phi_{0}^{\text {scat }}=-\left.\phi_{0}^{\text {inc }}\right|_{\partial D_{j}}+V_{j}
$$

where $V_{j}$ is an arbitrary constant on $\partial D_{j}$ that represents the voltage of each conductor (with respect to infinity). The Dirichlet field $\mathbf{Y}_{j}$ corresponds to the gradient of $\phi_{0}^{\text {scat }}$ obtained by setting $\phi_{0}^{\text {scat }}$ to 0 on each boundary component $\partial D_{i}$ for $i \neq j$ and $\phi_{0}^{\text {scat }}=1$ on $\partial D_{j}$.

Let us now define the scalars $Q_{j}$ by

$$
Q_{j}=\int_{\partial D_{j}} \frac{\partial \phi_{0}^{\mathrm{scat}}}{\partial n} d s=-\int_{\partial D_{j}} \mathbf{n} \cdot \mathbf{E}_{0}^{\mathrm{scat}} d s,
$$

so that $-Q_{j}$ is the total charge on each conductor $\partial D_{j}$. The matrix that links the voltages $V_{j}$ and the charges $-Q_{j}$ is known as the capacitance matrix [17]. Since we are interested here in the time-harmonic Maxwell equations and their zero-frequency limit, we must have charge neutrality on each boundary component. Thus, we are interested in studying (2.5) where the voltages $V_{j}$ are additional unknowns, but for which $N$ additional constraints are given of the form $Q_{j}=0$ for $j=1, \ldots, N$.

It is important to note that, in the static regime, the problem suffers from more than nonuniqueness. The boundary condition

$$
\boldsymbol{n} \times \nabla \phi_{0}^{\text {scat }}=-\boldsymbol{n} \times \boldsymbol{E}_{0}^{\mathrm{inc}}
$$

cannot be satisfied unless the incoming field is also an electrostatic field. In particular, if the circulation of the incoming field $\oint_{L \subset \partial D} \boldsymbol{E}_{0}^{\text {inc }} \cdot d \mathbf{l}$ is not zero on every closed loop $L$ on the surface $\partial D$, the solution $\boldsymbol{E}_{0}^{\text {scat }}$ does not exist. This follows easily from the fact that

$$
\oint_{L \subset \partial D} \boldsymbol{E}_{0}^{\mathrm{inc}} \cdot d \mathbf{l}=-\oint_{L \subset \partial D} \nabla \phi_{0}^{\mathrm{scat}} \cdot d \mathbf{l}=0 .
$$

We refer the reader to [10] for further discussion. 


\subsection{Magnetostatics}

The magnetostatic field satisfies the equations

$$
\nabla \times \boldsymbol{H}_{0}(\boldsymbol{x})=0, \quad \nabla \cdot \boldsymbol{H}_{0}(\boldsymbol{x})=0, \quad \boldsymbol{x} \in \mathbb{R}^{3} / D,
$$

and the boundary condition

$$
\boldsymbol{n} \cdot \boldsymbol{H}_{0}(\boldsymbol{x})=\left.0\right|_{\partial D}
$$

The total field is again decomposed into incoming and scattered fields, with the scattered field satisfying the radiation condition (2.9),

$$
\boldsymbol{H}_{0}^{\text {scat }}(\boldsymbol{x})=o(1), \quad|\boldsymbol{x}| \rightarrow \infty .
$$

$\boldsymbol{H}_{0}^{\text {scat }}$ can be described either as the curl of a harmonic vector potential $\mathbf{A}_{\mathbf{0}}$ or as the gradient of a harmonic scalar potential $\phi_{0}^{\text {scat }}$.

The magnetostatic problem also suffers from nonuniqueness. The nullspace, that is functions satisfying (2.7) and (2.8) and the radiation condition (2.9), is of dimension $g$, where $g$ is the genus of the surface $\partial D$. Elements of this space are called harmonic Neumann fields $\left\{\mathbf{Z}_{m}\right\}_{m=1}^{g}[10]$. In order to completely specify the solution, additional information, such as the total induced current $I_{m}$ on $g$ loops lying on the surface, must be specified:

$$
\oint_{l_{m}} \boldsymbol{H}_{0}^{\mathrm{scat}}(\boldsymbol{x}) \cdot d \mathbf{l}=I_{m}, \quad m=1, \ldots, g .
$$

The loops $l_{m}$ here go around the "holes" (see Figure 1.1). That is, they are a basis for the first homology group of $\mathbb{R}^{3} \backslash D$, with spanning surfaces that lie in the interior of the scatterer; see [10] for more details. The persistent currents $I_{m}$ at zero frequency are due to the potential presence of superconducting loops (as we are considering scattering from perfect electric conductors).

\subsection{Summary}

To summarize, the problem of electromagnetic scattering from perfect conductors is uniquely solvable for any $\omega$ strictly greater than 0 . At $\omega=0$, however, various subtleties arise. The issue of Dirichlet fields needs to be resolved in electrostatics and the issue of Neumann fields needs to be resolved in magnetostatics. For any $\omega$ strictly greater than 0 , however, it is necessary that the total charge $Q_{j}$ induced on any connected component of the scatterer be 0 . Enforcing this condition at $\omega=0$ (and introducing the additional unknown constants $V_{j}$ as above) uniquely determines the electrostatic field. In the magnetostatic case, however, we are obligated to introduce additional constants, such as the $\left\{I_{m}\right\}$ in 2.10, in order to account for the Neumann fields when the scatterer has nonzero genus [25]. 


\section{Scattering Theory for Decoupled Potentials}

We turn now to the analytic foundations of the DPIE. We first derive boundary value problems for the scattered scalar and vector potentials that are completely insensitive to the genus, although they do depend explicitly on the number of boundary components. After this reformulation of the Maxwell equations, we design integral representations that lead to well-conditioned and invertible linear systems of equations.

For the sake of simplicity we will assume in the rest of the paper that $D$ is a bounded open region in $\mathbb{R}^{3}$. The boundary of $D$, denoted by $\partial D$, is assumed to consist of a finite number of disjoint, closed bounded surfaces belonging to class $C^{2}$, and the exterior region $\mathbb{R}^{3} \backslash D$ is assumed to be connected.

DEFINITION 3.1. By the scalar Dirichlet problem, we mean the calculation of a scalar Helmholtz or Laplace potential $\phi^{\text {scat }} \in C^{2}\left(\mathbb{R}^{3} \backslash \bar{D}\right) \cap C\left(\mathbb{R}^{3} \backslash D\right)$ whose boundary value equals a given continuous function $f \in C(\partial D)$ and which satisfies standard radiation conditions at infinity:

$$
\begin{gathered}
\Delta \phi^{\text {scat }}+k^{2} \phi^{\text {scat }}=0,\left.\quad \phi^{\text {scat }}\right|_{\partial D}=f, \\
\frac{\boldsymbol{x}}{|\boldsymbol{x}|} \cdot \nabla \phi^{\text {scat }}(\boldsymbol{x})-i k \phi^{\text {scat }}(\boldsymbol{x})=o\left(\frac{1}{|\boldsymbol{x}|}\right), \quad|\boldsymbol{x}| \rightarrow \infty
\end{gathered}
$$

(uniformly for all directions $\frac{\boldsymbol{x}}{|\boldsymbol{x}|}$ ) for the scalar Helmholtz potential, and

$$
\begin{gathered}
\Delta \phi_{0}^{\text {scat }}=0,\left.\quad \phi_{0}^{\text {scat }}\right|_{\partial D}=f, \\
\phi_{0}^{\text {scat }}(x)=o(1), \quad|x| \rightarrow \infty,
\end{gathered}
$$

(uniformly for all directions $\frac{\boldsymbol{x}}{|\boldsymbol{x}|}$ ) for the scalar Laplace potential, respectively.

DEFINITION 3.2. By the vector Dirichlet problem, we mean the calculation of a vector Helmholtz or Laplace potential $\boldsymbol{A}^{\text {scat }} \in C^{2}\left(\mathbb{R}^{3} \backslash \bar{D}\right)$ with $\nabla \cdot \boldsymbol{A}^{\text {scat }} \in$ $C\left(\mathbb{R}^{3} \backslash D\right)$ whose tangential boundary values equal a given continuous tangential function $\mathbf{f} \in C_{t}(\partial D)$, whose divergence equals a given scalar continuous function $h \in C(\partial D)$ and which satisfies standard radiation conditions at infinity:

$$
\begin{gathered}
\Delta \boldsymbol{A}^{\text {scat }}+k^{2} \boldsymbol{A}^{\text {scat }}=0, \quad \boldsymbol{n} \times\left.\boldsymbol{A}^{\text {scat }}\right|_{\partial D^{+}}=\mathbf{f},\left.\quad \nabla \cdot \boldsymbol{A}^{\text {scat }}\right|_{\partial D}=h, \\
\nabla \times \boldsymbol{A}^{\text {scat }}(\boldsymbol{x}) \times \frac{\boldsymbol{x}}{|\boldsymbol{x}|}+\frac{\boldsymbol{x}}{|\boldsymbol{x}|} \nabla \cdot \boldsymbol{A}^{\mathrm{scat}}(\boldsymbol{x})-i k \boldsymbol{A}^{\mathrm{scat}}(\boldsymbol{x})=o\left(\frac{1}{|\boldsymbol{x}|}\right), \\
|\boldsymbol{x}| \rightarrow \infty
\end{gathered}
$$

(uniformly for all directions $\frac{\boldsymbol{x}}{|\boldsymbol{x}|}$ ) for the vector Helmholtz potential, and

$$
\begin{gathered}
\Delta \boldsymbol{A}_{0}^{\text {scat }}=0, \quad \boldsymbol{n} \times\left.\boldsymbol{A}_{0}^{\text {scat }}\right|_{\partial D^{+}}=\mathbf{f},\left.\quad \nabla \cdot \boldsymbol{A}_{0}^{\text {scat }}\right|_{\partial D}=h, \\
\boldsymbol{A}_{0}^{\text {scat }}(\boldsymbol{x})=o(1), \quad|\boldsymbol{x}| \rightarrow \infty
\end{gathered}
$$

(uniformly for all directions $\frac{\boldsymbol{x}}{|\boldsymbol{x}|}$ ) for the vector Laplace potential. 


\begin{tabular}{|c|c|c|}
\hline $\begin{array}{l}\text { Unknowns } \\
\phi^{\text {scat }}, \boldsymbol{A}^{\text {scat }}\end{array}$ & Laplace & Helmholtz \\
\hline Scalar & $\left\{\begin{array}{l}\Delta \phi^{\text {scat }}=0 \\
\left.\phi^{\text {scat }}\right|_{\partial D}=f .\end{array}\right.$ & $\left\{\begin{array}{l}\Delta \phi^{\text {scat }}+k^{2} \phi^{\text {scat }}=0 \\
\left.\phi^{\text {scat }}\right|_{\partial D}=f\end{array}\right.$ \\
\hline Vector & $\left\{\begin{array}{l}\Delta \boldsymbol{A}^{\text {scat }}=0, \\
\boldsymbol{n} \times\left.\boldsymbol{A}^{\text {scat }}\right|_{\partial D^{+}}=\mathbf{f}, \quad(\mathrm{No}) \\
\left.\nabla \cdot \boldsymbol{A}^{\text {scat }}\right|_{\partial D}=h .\end{array}\right.$ & $\left\{\begin{array}{l}\Delta \boldsymbol{A}^{\text {scat }}+k^{2} \boldsymbol{A}^{\text {scat }}=0, \\
\left.\boldsymbol{n} \times\left.\boldsymbol{A}^{\text {scat }}\right|_{\partial D^{+}}=\mathbf{f}, \quad \text { (Yes }\right) \\
\left.\nabla \cdot \boldsymbol{A}^{\text {scat }}\right|_{\partial D}=h .\end{array}\right.$ \\
\hline
\end{tabular}

TABLE 3.1. Uniqueness for Dirichlet problems

The boundary condition on the tangential components of $\boldsymbol{A}^{\text {scat }}$ should be understood in the sense

$$
\boldsymbol{n} \times\left.\boldsymbol{A}^{\text {scat }}\right|_{\partial D^{+}}:=\lim _{\delta \rightarrow 0+} \boldsymbol{n}(\mathbf{x}) \times \boldsymbol{A}^{\text {scat }}(\mathbf{x}+\delta \boldsymbol{n}(\mathbf{x}))
$$

where the limit must exist uniformly for all $\mathbf{x} \in \partial D$. Note that the normal component of $\boldsymbol{A}^{\text {scat }}$ might not be continuous up to the boundary in the same sense as the tangential components.

For $k \neq 0$, both Dirichlet problems have unique solutions, but for $k=0$, the vector Dirichlet problem has a nullspace — the harmonic Dirichlet fields discussed in Section 2.1. This lack of uniqueness also makes the vector Dirichlet problem ill-conditioned at low frequencies.

\subsection{Modified Dirichlet Problems}

In order to address the nonuniqueness of the vector Dirichlet problem at zero frequency and in order to enforce that the uncoupled scalar and vector potentials define a suitable Maxwell field (enforcing the Lorenz gauge), we introduce a related set of boundary value problems, which we refer to as the modified Dirichlet problems.

Definition 3.3. By the scalar modified Dirichlet problem, we mean the calculation of a scalar Helmholtz or Laplace potential $\phi^{\text {scat }} \in C^{2}\left(\mathbb{R}^{3} \backslash \bar{D}\right) \cap C\left(\mathbb{R}^{3} \backslash D\right)$ that satisfies standard radiation conditions at infinity, and a set of constants $\left\{V_{j}\right\}_{j=1}^{N}$, where $N$ denotes the number of connected components of the boundary $\partial D$. Given boundary data $f \in C(\partial D)$ and known constants $\left\{Q_{j}\right\}_{j=1}^{N}$, the scalar Helmholtz potential and the $\left\{V_{j}\right\}_{j=1}^{N}$ must satisfy

$$
\begin{gathered}
\Delta \phi^{\text {scat }}+k^{2} \phi^{\text {scat }}=0,\left.\quad \phi^{\text {scat }}\right|_{\partial D_{j}}=f+V_{j}, \\
\frac{\boldsymbol{x}}{|\boldsymbol{x}|} \cdot \nabla \phi^{\text {scat }}(\boldsymbol{x})-i k \phi^{\text {scat }}(\boldsymbol{x})=o\left(\frac{1}{|\boldsymbol{x}|}\right), \quad|\boldsymbol{x}| \rightarrow \infty
\end{gathered}
$$


(uniformly for all directions $\frac{\boldsymbol{x}}{|\boldsymbol{x}|}$ ), with the flux condition

$$
\int_{\partial D_{j}^{+}} \frac{\partial \phi^{\text {scat }}}{\partial n} d s=Q_{j}
$$

The scalar Laplace potential and the constants $\left\{V_{j}\right\}_{j=1}^{N}$ must satisfy

$$
\begin{gathered}
\Delta \phi_{0}^{\text {scat }}=0,\left.\quad \phi_{0}^{\text {scat }}\right|_{\partial D_{j}}=f+V_{j}, \\
\phi_{0}^{\text {scat }}(\boldsymbol{x})=o(1), \quad|\boldsymbol{x}| \rightarrow \infty
\end{gathered}
$$

(uniformly for all directions $\frac{\boldsymbol{x}}{|\boldsymbol{x}|}$ ), with the same flux condition (3.12),

$$
\int_{\partial D_{j}^{+}} \frac{\partial \phi_{0}^{\text {scat }}}{\partial n} d s=Q_{j}
$$

The flux condition 3.12 must be interpreted as

$$
\int_{\partial D_{j}^{+}} \frac{\partial \phi^{\text {scat }}}{\partial n} d s:=\lim _{\delta \rightarrow 0^{+}} \int_{\partial D_{j}^{\delta}} \frac{\partial \phi^{\text {scat }}}{\partial n} d s=Q_{j} .
$$

See Appendix A for further details.

Definition 3.4. By the vector modified Dirichlet problem, we mean the calculation of a vector Helmholtz or Laplace potential $A^{\text {scat }} \in C^{2}\left(\mathbb{R}^{3} \backslash \bar{D}\right)$ with $\nabla \cdot \boldsymbol{A}^{\text {scat }} \in C\left(\mathbb{R}^{3} \backslash D\right)$ that satisfies standard radiation conditions at infinity, and a set of constants $\left\{v_{j}\right\}_{j=1}^{N}$, where $N$ denotes the number of connected components of the boundary $\partial D$. Given tangential boundary data $\mathbf{f} \in C_{t}(\partial D)$, scalar boundary data $h \in C(\partial D)$, and known constants $\left\{q_{j}\right\}_{j=1}^{N}$, the vector Helmholtz potential and the constants $\left\{v_{j}\right\}_{j=1}^{N}$ must satisfy

$$
\begin{gathered}
\Delta \boldsymbol{A}^{\text {scat }}+k^{2} \boldsymbol{A}^{\text {scat }}=0, \quad \boldsymbol{n} \times\left.\boldsymbol{A}^{\text {scat }}\right|_{\partial D^{+}}=\mathbf{f},\left.\quad \nabla \cdot \boldsymbol{A}^{\text {scat }}\right|_{\partial D_{j}}=h+v_{j}, \\
\nabla \times \boldsymbol{A}^{\text {scat }}(\boldsymbol{x}) \times \frac{\boldsymbol{x}}{|\boldsymbol{x}|}+\frac{\boldsymbol{x}}{|\boldsymbol{x}|} \nabla \cdot \boldsymbol{A}^{\text {scat }}(\boldsymbol{x})-i k \boldsymbol{A}^{\text {scat }}(\boldsymbol{x})=o\left(\frac{1}{|\boldsymbol{x}|}\right) \\
|\boldsymbol{x}| \rightarrow \infty
\end{gathered}
$$

with

$$
\int_{\partial D_{j}^{+}} \boldsymbol{n} \cdot \boldsymbol{A}^{\text {scat }} d s=q_{j} .
$$

The vector Laplace potential and the constants $\left\{v_{j}\right\}_{j=1}^{N}$ must satisfy

$$
\begin{gathered}
\Delta \boldsymbol{A}_{0}^{\text {scat }}=0, \quad \boldsymbol{n} \times\left.\boldsymbol{A}_{0}^{\text {scat }}\right|_{\partial D^{+}}=\mathbf{f},\left.\quad \nabla \cdot \boldsymbol{A}_{0}^{\text {scat }}\right|_{\partial D_{j}}=h+v_{j}, \\
\boldsymbol{A}_{0}^{\text {scat }}(\boldsymbol{x})=o(1), \quad|\boldsymbol{x}| \rightarrow \infty
\end{gathered}
$$




\begin{tabular}{|c|c|c|}
\hline $\begin{array}{l}\text { Unknowns } \\
\phi^{\text {scat }},\left\{V_{j}\right\}_{j=1}^{N} \\
\boldsymbol{A}^{\text {scat }},\left\{v_{j}\right\}_{j=1}^{N}\end{array}$ & Laplace & Helmholtz \\
\hline Scalar & $\left\{\begin{array}{l}\Delta \phi^{\text {scat }}=0, \\
\left.\phi^{\text {scat }}\right|_{\partial D_{j}}=f+V_{j}, \quad \text { (Yes) } \\
\int_{\partial D_{j}^{+}} \frac{\partial \phi^{\text {scat }}}{\partial n} d s=Q_{j} .\end{array}\right.$ & $\left\{\begin{array}{l}\Delta \phi^{\text {scat }}+k^{2} \phi^{\text {scat }}=0 \\
\left.\phi^{\text {scat }}\right|_{\partial D_{j}}=f+V_{j} \\
\int_{\partial D_{j}^{+}} \frac{\partial \phi^{\text {scat }}}{\partial n} d s=Q_{j}\end{array}\right.$ \\
\hline Vector & $\left\{\begin{array}{l}\Delta \boldsymbol{A}^{\text {scat }}=0, \\
\boldsymbol{n} \times\left.\boldsymbol{A}^{\text {scat }}\right|_{\partial D^{+}}=\mathbf{f}, \\
\left.\nabla \cdot \boldsymbol{A}^{\text {scat }}\right|_{\partial D_{j}}=h+v_{j}, \quad(\text { Yes }) \\
\int_{\partial D_{j}^{+}} \boldsymbol{n} \cdot \boldsymbol{A}^{\text {scat }} d s=q_{j} .\end{array}\right.$ & $\left\{\begin{array}{l}\Delta \boldsymbol{A}^{\text {scat }}+k^{2} \boldsymbol{A}^{\text {scat }}=0, \\
\boldsymbol{n} \times\left.\boldsymbol{A}^{\text {scat }}\right|_{\partial D^{+}}=\mathbf{f}, \\
\left.\nabla \cdot \boldsymbol{A}^{\text {scat }}\right|_{\partial D_{j}}=h+v_{j}, \quad(\text { Yes }) \\
\int_{\partial D_{j}^{+}} \boldsymbol{n} \cdot \boldsymbol{A}^{\text {scat }} d s=q_{j}\end{array}\right.$ \\
\hline
\end{tabular}

TABLE 3.2. Uniqueness for modified Dirichlet problems.

(uniformly for all directions $\frac{\boldsymbol{x}}{|\boldsymbol{x}|}$ ), with the same flux condition (3.17).

We summarize the modified Dirichlet boundary value problems in Table 3.2 .

We now define the scattered scalar and vector potentials in terms of modified Dirichlet problems.

Definition 3.5. Let $\phi^{\text {inc }}, A^{\text {inc }}$ denote incoming scalar and vector potentials and assume that $D$ is a perfect conductor. The scattered scalar potential $\phi^{\text {scat }}$ is the solution to the scalar modified Dirichlet problem with boundary data:

$$
f:=-\left.\phi^{\mathrm{inc}}\right|_{\partial D_{j}}, \quad Q_{j}:=-\int_{\partial D_{j}} \frac{\partial \phi^{\mathrm{inc}}}{\partial n} d s .
$$

Likewise, the scattered vector potential $\boldsymbol{A}^{\text {scat }}$ is the solution to the vector modified Dirichlet problem with boundary data:

$$
\mathbf{f}:=-\boldsymbol{n} \times\left.\boldsymbol{A}^{\mathrm{inc}}\right|_{\partial D}, \quad h:=-\left.\nabla \cdot \boldsymbol{A}^{\mathrm{inc}}\right|_{\partial D}, \quad q_{j}:=-\int_{\partial D_{j}} \boldsymbol{n} \cdot \boldsymbol{A}^{\mathrm{inc}} d s .
$$

Next we prove a lemma that shows the connection between the scalar and vector modified Dirichlet problems. For simplicity, in the remainder of the paper we assume that $\epsilon>0, \mu>0$, so that $k$ is real and nonnegative. The proofs are analogous when the wavenumber $k$ has a positive imaginary part, which adds dissipation.

LEMMA 3.6. Let $\boldsymbol{A}^{\text {scat }},\left\{v_{j}\right\}_{j=1}^{N}$ be a solution of the vector modified Dirichlet problem with boundary data $\mathbf{f}, h,\left\{q_{j}\right\}_{j=1}^{N}$ for $k \geq 0$. Then,

$$
\psi^{\text {scat }}:=\nabla \cdot \boldsymbol{A}^{\text {scat }}, \quad\left\{V_{j}=v_{j}\right\}_{j=1}^{N},
$$

satisfies the scalar modified Dirichlet problem with boundary data:

$$
f:=h, \quad\left\{Q_{j}=-k^{2} q_{j}\right\}_{j=1}^{N} .
$$


Proof. By hypothesis we have that $\psi^{\text {scat }}:=\nabla \cdot A^{\text {scat }} \in C\left(\mathbb{R}^{3} \backslash D\right)$. We also have that $\boldsymbol{A}^{\text {scat }}$ satisfies

$$
\begin{gathered}
\Delta \boldsymbol{A}^{\text {scat }}+k^{2} \boldsymbol{A}^{\text {scat }}=0, \\
\boldsymbol{n} \times\left.\boldsymbol{A}^{\text {scat }}\right|_{\partial D^{+}}=\mathbf{f} \\
\left.\nabla \cdot \boldsymbol{A}^{\text {scat }}\right|_{\partial D_{j}}=h+v_{j}, \\
\int_{\partial D_{j}^{+}} \boldsymbol{n} \cdot \boldsymbol{A}^{\text {scat }} d s=q_{j} .
\end{gathered}
$$

Taking the divergence of (3.24), we get

$$
\Delta \nabla \cdot \boldsymbol{A}^{\text {scat }}+k^{2} \nabla \cdot \boldsymbol{A}^{\text {scat }}=0 .
$$

Therefore, $\psi^{\text {scat }}:=\nabla \cdot A^{\text {scat }}$ satisfies the Helmholtz equation. From 3.26 , we get

$$
\psi^{\text {scat }}=\nabla \cdot \boldsymbol{A}^{\text {scat }}=h+\left.v_{j}\right|_{\partial D_{j}} .
$$

Notice that $\boldsymbol{A}^{\text {scat }}$ is a Helmholtz (or Laplace) potential, analytic at every exterior point, so that $\psi^{\text {scat }}:=\nabla \cdot A^{\text {scat }} \in C^{2}\left(\mathbb{R}^{3} \backslash \bar{D}\right) \cap C\left(\mathbb{R}^{3} \backslash D\right)$. Finally, we may write

$$
\nabla \times \nabla \times \boldsymbol{A}^{\text {scat }}=k^{2} \boldsymbol{A}^{\text {scat }}+\nabla \nabla \cdot \boldsymbol{A}^{\text {scat }}
$$

for any point $\boldsymbol{x} \in \mathbb{R}^{3} \backslash \bar{D}$. Taking the flux on a parallel surface $\partial D_{j}^{\delta}$ for $h$ small enough we have

$$
\begin{aligned}
\int_{\partial D_{j}^{\delta}} \boldsymbol{n} \cdot \nabla \times \nabla \times \boldsymbol{A}^{\text {scat }} d s & =\int_{\partial D_{j}^{\delta}} k^{2} \boldsymbol{n} \cdot \boldsymbol{A}^{\text {scat }}+\boldsymbol{n} \cdot \nabla \nabla \cdot \boldsymbol{A}^{\text {scat }} d s, \\
\int_{\partial D_{j}^{\delta}}-\nabla_{s} \cdot\left(\boldsymbol{n} \times \nabla \times \boldsymbol{A}^{\mathrm{scat}}\right) d s & =k^{2} \int_{\partial D_{j}^{\delta}} \boldsymbol{n} \cdot \boldsymbol{A}^{\text {scat }} d s+\int_{\partial D_{j}^{\delta}} \boldsymbol{n} \cdot \nabla \frac{\partial \psi^{\mathrm{scat}}}{\partial n} d s,
\end{aligned}
$$

so that

$$
k^{2} \int_{\partial D_{j}^{\delta}} \boldsymbol{n} \cdot \boldsymbol{A}^{\mathrm{scat}} d s+\int_{\partial D_{j}^{\delta}} \frac{\partial \psi^{\mathrm{scat}}}{\partial n} d s=0 .
$$

Taking the limit for $\delta \rightarrow 0$ and using the boundary condition 3.27), we obtain

$$
\int_{\partial D_{j}^{+}} \frac{\partial \psi^{\text {scat }}}{\partial n} d s=-k^{2} q_{j},
$$

and the desired result follows. 


\subsection{Uniqueness}

We begin with two well-known theorems from scattering theory.

THEOREM $3.7([10])$. Let $\phi^{\text {scat }} \in C^{2}\left(\mathbb{R}^{3} \backslash \bar{D}\right) \cap C\left(\mathbb{R}^{3} \backslash D\right)$ be a scalar Helmholtz potential in the exterior domain possessing normal derivatives in the sense of uniform convergence (see (A.1)) with wavenumber $k, k \neq 0$, in the exterior domain $\mathbb{R}^{3} \backslash \bar{D}$ satisfying the radiation condition (3.2) and the condition

$$
I_{s}=\operatorname{Im}\left\{k \int_{\partial D} \phi^{\text {scat }} \frac{\partial \bar{\phi}^{\text {scat }}}{\partial n} d s\right\} \geq 0,
$$

where $\operatorname{Im}\{f\}$ denotes the imaginary part of $f$. Then $\phi^{\text {scat }}=0$ in $\mathbb{R}^{3} \backslash D$.

THEOREM $3.8([10])$. Let $\boldsymbol{A}^{\text {scat }} \in C^{2}\left(\mathbb{R}^{3} \backslash \bar{D}\right) \cap C\left(\mathbb{R}^{3} \backslash D\right)$ be a vector Helmholtz potential in the exterior domain with $\nabla \cdot \boldsymbol{A}^{\text {scat }}, \nabla \times \boldsymbol{A}^{\text {scat }} \in C\left(\mathbb{R}^{3} \backslash D\right)$, with wavenumber $k, k \neq 0$, satisfying the radiation condition (3.6) and the condition

$$
I_{v}=\operatorname{Im}\left\{k \int_{\partial D} \boldsymbol{n} \times \boldsymbol{A}^{\text {scat }} \cdot \nabla \times \overline{\boldsymbol{A}}^{\text {scat }}+\boldsymbol{n} \cdot \boldsymbol{A}^{\text {scat }} \nabla \cdot \overline{\boldsymbol{A}}^{\text {scat }} d s\right\} \geq 0 .
$$

Then, $\boldsymbol{A}^{\text {scat }}=0$ in $\mathbb{R}^{3} \backslash D$.

We now show that the modified Dirichlet problems have unique solutions in all regimes.

THEOREM 3.9. The scalar modified Dirichlet problem has at most one solution for any $k>0$.

PROOF. Consider a solution of the homogeneous problem $\left(f=0, Q_{j}=0\right)$

$$
\left\{\begin{array}{l}
\Delta \phi^{\text {scat }}+k^{2} \phi^{\text {scat }}=0 \\
\left.\phi^{\text {scat }}\right|_{\partial D}=0+V_{j} \\
\int_{\partial D_{j}^{+}} \frac{\partial \phi^{\text {scat }}}{\partial n} d s=0 .
\end{array}\right.
$$

If $\phi^{\text {scat }}$ had a normal derivative in the sense of uniform convergence (see A.1, we could immediately apply Theorem 3.7. However, in our formulation of the scalar modified Dirichlet problem, we require $\phi^{\text {scat }}$ only to be continuous up to the boundary. There are several approaches to overcoming this difficulty. Convergence theorems for Lebesgue integration could be used, as in lemma 3.10 of [11]. Here, we simply use the fact that the function $\left.\phi^{\text {scat }}\right|_{\partial D}$ is constant on each connected component $\partial D_{j}$ of $\partial D$. Thus, $\left.\phi^{\text {scat }}\right|_{\partial D} \in C^{1, \alpha}(\partial D)$. Applying theorem 3.27 of [10], we have that the solution $\phi^{\text {scat }} \in C^{1, \alpha}\left(\mathbb{R}^{3} \backslash D\right)$, and we can apply Theorem 3.7. The quantity $I_{s}$ in 3.32) is then given by

$$
I_{s}=\operatorname{Im}\left\{k \int_{\partial D} \phi^{\text {scat }} \frac{\partial \bar{\phi}^{\text {scat }}}{\partial n} d s\right\}=\operatorname{Im}\left\{k \sum_{j=1}^{N} V_{j} \int_{\partial D_{j}} \frac{\partial \bar{\phi}^{\text {scat }}}{\partial n}\right\}=0 .
$$


Thus, by Theorem 3.7, $\phi^{\text {scat }}=0$ and using the boundary condition $\left.\phi^{\text {scat }}\right|_{\partial D_{j}}=$ $0+V_{j}$, we get $V_{j}=0$.

THEOREM 3.10. The vector modified Dirichlet problem has at most one solution for any $k>0$.

PROOF. Consider a solution of the homogeneous problem ( $f=0, h=0$, $\left.q_{j}=0\right)$ :

$$
\left\{\begin{array}{l}
\Delta \boldsymbol{A}^{\text {scat }}+k^{2} \boldsymbol{A}^{\text {scat }}=0, \\
\boldsymbol{n} \times\left.\boldsymbol{A}^{\text {scat }}\right|_{\partial D^{+}}=\mathbf{0}, \\
\left.\nabla \cdot \boldsymbol{A}^{\text {scat }}\right|_{\partial D_{j}}=0+v_{j}, \\
\int_{\partial D_{j}^{+}} \boldsymbol{n} \cdot \boldsymbol{A}^{\text {scat }} d s=0 .
\end{array}\right.
$$

Again, in order to be able to use Theorem 3.8 we need continuity of the function $\boldsymbol{A}^{\text {scat }}$ and its derivatives up to the boundary. As before, we can use the fact that the regularity of the boundary data in the homogeneous case implies the regularity of the solution up to the boundary. The technique of [8,16], could be used, requiring some technical arguments concerning Hölder space analysis on surfaces parallel to $\partial D_{\delta}$.

A simpler proof is obtained by applying Lemma 3.6, which shows that $\nabla \cdot \boldsymbol{A}^{\text {scat }}$ satisfies the homogeneous modified Dirichlet problem. By Theorem 3.9 we have that $\nabla \cdot \boldsymbol{A}^{\text {scat }}(\mathbf{x})=0$ for $\mathbf{x} \in \mathbb{R}^{3} \backslash D$. Let us now define the vector fields

$$
\breve{\boldsymbol{E}}:=\boldsymbol{A}^{\text {scat }}, \quad \breve{\boldsymbol{H}}:=\frac{1}{i \omega \mu} \nabla \times \boldsymbol{A}^{\text {scat }} .
$$

These fields satisfy the exterior homogeneous Maxwell problem and $\breve{\boldsymbol{E}}, \breve{\boldsymbol{H}} \in$ $C^{1}\left(\mathbb{R}^{3} \backslash \bar{D}\right)$. Applying theorem 6.23 of $[11]$ we have that $\boldsymbol{A}^{\text {scat }}(\mathbf{x})=0$ for $\mathbf{x} \in \mathbb{R}^{3} \backslash D$.

Using the boundary condition $\left.\nabla \cdot \boldsymbol{A}^{\text {scat }}\right|_{\partial D_{j}}=0+v_{j}$, we obtain $v_{j}=0$.

THEOREM 3.11. The scalar modified Dirichlet problem for the Laplace equation has at most one solution.

Proof. As in Theorem 3.9, we have that the normal derivative of $\phi_{0}^{\text {scat }}$ exists in a strong sense if $\phi_{0}^{\text {scat }}$ satisfies the homogeneous scalar modified Dirichlet problem. Note also that the radiation condition implies a strong decay at infinity (see (5.1) in [10]). This permits the application of Green's theorem in the exterior region from which

$$
\int_{\mathbb{R}^{3} \backslash \bar{D}}\left|\nabla \phi_{0}^{\text {scat }}\right|^{2} d v=\int_{\partial D} \phi_{0}^{\text {scat }} \frac{\partial \bar{\phi}_{0}^{\text {scat }}}{\partial n} d s=\sum_{j=1}^{N} V_{j} \int_{\partial D_{j}} \frac{\partial \bar{\phi}_{0}^{\text {scat }}}{\partial n} d s=0 .
$$

Thus, $\nabla \phi_{0}^{\text {scat }}=0$ in the exterior domain. Using the radiation condition, we obtain $\phi_{0}^{\text {scat }}=0$. Using the boundary condition $\left.\phi_{0}^{\text {scat }}\right|_{\partial D_{j}}=0+V_{j}$, we have $V_{j}=0$. 
THEOREM 3.12. The vector modified Dirichlet problem for the Laplace equation has at most one solution.

Proof. Let $\left(\boldsymbol{A}^{\text {scat }}, v_{j}\right)$ be a solution of the homogeneous vector modified Dirichlet problem. Again, we can assume the regularity of the homogeneous solution up to the boundary and apply Green's theorem as in Theorem 3.11 . We then have

$$
\begin{aligned}
& \int_{\mathbb{R}^{3 \backslash \bar{D}}}\left|\nabla \times \boldsymbol{A}_{0}^{\text {scat }}\right|^{2}+\left|\nabla \cdot \boldsymbol{A}_{0}^{\text {scat }}\right| d v \\
& \quad=\int_{\partial D} \boldsymbol{n} \times \boldsymbol{A}_{0}^{\text {scat }} \cdot \nabla \times \overline{\boldsymbol{A}}_{0}^{\text {scat }}+\boldsymbol{n} \cdot \boldsymbol{A}_{0}^{\text {scat }} \nabla \overline{\boldsymbol{A}}_{0}^{\text {scat }} d s \\
& \quad=\sum_{j=1}^{N} \bar{v}_{j} \int_{\partial D_{j}} \boldsymbol{n} \cdot \boldsymbol{A}^{\text {scat }} d s=0 .
\end{aligned}
$$

Thus, $\nabla \times \boldsymbol{A}_{0}^{\text {scat }}=0$ and $\nabla \cdot \boldsymbol{A}_{0}^{\text {scat }}=0$ in the exterior region, and $\boldsymbol{A}_{0}^{\text {scat }}$ is a harmonic field in $\mathbb{R}^{3} \backslash \bar{D}$. From the boundary condition $\boldsymbol{n} \times \boldsymbol{A}_{0}^{\text {scat }}=\left.0\right|_{\partial D}$, we have that $\boldsymbol{A}_{0}^{\text {scat }}$ is a Dirichlet field and thus a linear combination of the basis functions $\left\{\mathbf{Y}_{j}\right\}_{j=1}^{N}$. It follows from the flux conditions $\int_{\partial D_{j}} \boldsymbol{n} \cdot \boldsymbol{A}^{\text {scat }} d s=0$ that $\boldsymbol{A}^{\text {scat }}=0$.

\subsection{Existence and Stability}

In this section, we use the Fredholm alternative to obtain existence results for the modified Dirichlet problems, making use of the single- and double-layer potentials, $S_{k}$ and $D_{k}$, of classical potential theory. We also show that the solution depends continuously on the boundary data, uniformly in $k$ in a neighborhood of $k=0$. Next we define classical operators in potential theory:

$$
\begin{array}{ll}
S_{k} \sigma=\int_{\partial D} g_{k}(\boldsymbol{x}-\boldsymbol{y}) \sigma(\boldsymbol{y}) d A_{\boldsymbol{y}}, & D_{k} \sigma=\int_{\partial D} \frac{\partial g_{k}}{\partial n_{y}}(\boldsymbol{x}-\boldsymbol{y}) \sigma(\boldsymbol{y}) d A_{\boldsymbol{y}}, \\
S_{k}^{\prime} \sigma=\int_{\partial D} \frac{\partial g_{k}}{\partial n_{x}}(\boldsymbol{x}-\boldsymbol{y}) \sigma(\boldsymbol{y}) d A_{\boldsymbol{y}}, & D_{k}^{\prime} \sigma=\frac{\partial}{\partial n_{x}} \int_{\partial D} \frac{\partial g_{k}}{\partial n_{y}}(\boldsymbol{x}-\boldsymbol{y}) \sigma(\boldsymbol{y}) d A_{\boldsymbol{y}},
\end{array}
$$

where $\boldsymbol{x} \in \partial D$ and the Green's function on the free space is

$$
g_{k}(\boldsymbol{x})=\frac{e^{i k|\boldsymbol{x}|}}{4 \pi|\boldsymbol{x}|}
$$

For off-surface evaluations $\boldsymbol{x} \in \mathbb{R}^{3} \backslash \partial D$ we have

$$
\begin{aligned}
& S_{k}[\sigma](\boldsymbol{x})=\int_{\partial D} g_{k}(\boldsymbol{x}-\boldsymbol{y}) \sigma(\boldsymbol{y}) d A_{\boldsymbol{y}}, \\
& D_{k}[\sigma](\boldsymbol{x})=\int_{\partial D} \frac{\partial g_{k}}{\partial n_{y}}(\boldsymbol{x}-\boldsymbol{y}) \sigma(\boldsymbol{y}) d A_{\boldsymbol{y}} .
\end{aligned}
$$


THEOREM 3.13. The scalar field

$$
\phi^{\text {scat }}(\boldsymbol{x})=D_{k}[\sigma](\boldsymbol{x})-i \eta S_{k}[\sigma](\boldsymbol{x}),
$$

with scalar density $\sigma$ of class $C(\partial D)$ and $\eta>0$, and the constants $\left\{V_{j}\right\}_{j=1}^{N}$ solve the modified Dirichlet problem $(k \geq 0)$ provided $\sigma$ and $\left\{V_{j}\right\}_{j=1}^{N}$ solve the equations

$$
\begin{gathered}
\frac{\sigma}{2}+D_{k} \sigma-i \eta S_{k} \sigma-\sum_{j=1}^{N} V_{j} \chi_{j}=f, \\
\int_{\partial D_{j}}\left(\left(D_{k}^{\prime}-D_{0}^{\prime}\right) \sigma+i \eta \frac{\sigma}{2}-i \eta S_{k}^{\prime} \sigma\right) d s=Q_{j},
\end{gathered}
$$

where $\chi_{j}$ denotes the characteristic function for boundary $\partial D_{j}$. The resulting integral equation is of the second kind on $C(\partial D) \times \mathbb{C}^{N}$ and resonance-free.

Proof. See Appendix B.

In order to study the vector modified Dirichlet problem, we define the following dyadic operators:

$$
\overline{\bar{L}}\left(\begin{array}{l}
\mathbf{a} \\
\rho
\end{array}\right)=\left(\begin{array}{l}
L_{11} \mathbf{a}+L_{12} \rho \\
L_{21} \mathbf{a}+L_{22} \rho
\end{array}\right)
$$

where

$$
\begin{aligned}
& L_{11} \mathbf{a}=M_{k} \mathbf{a}=\int_{\partial D} \boldsymbol{n}_{x} \times \nabla_{x} \times\left(g_{k}(\boldsymbol{x}-\boldsymbol{y}) \mathbf{a}(\boldsymbol{y})\right) d A_{\boldsymbol{y}}, \\
& L_{12} \rho=-\boldsymbol{n} \times S_{k}(\boldsymbol{n} \rho), \quad L_{21} \mathbf{a}=0, \quad L_{22} \rho=D_{k} \rho,
\end{aligned}
$$

and

$$
\overline{\bar{R}}\left(\begin{array}{l}
\mathbf{a} \\
\rho
\end{array}\right)=\left(\begin{array}{l}
R_{11} \mathbf{a}+R_{12} \rho \\
R_{21} \mathbf{a}+R_{22} \rho
\end{array}\right)
$$

where

$$
\begin{array}{ll}
R_{11} \mathbf{a}=\boldsymbol{n} \times S_{k}(\boldsymbol{n} \times \mathbf{a}), & R_{12} \rho=\boldsymbol{n} \times \nabla S_{k}(\rho), \\
R_{21} \mathbf{a}=\nabla \cdot S_{k}(\boldsymbol{n} \times \mathbf{a}), & R_{22} \rho=-k^{2} S_{k} \rho .
\end{array}
$$

and

$$
\overline{\bar{S}}_{i k}\left(\begin{array}{c}
\mathbf{a} \\
\rho
\end{array}\right)=\left(\begin{array}{l}
S_{i k}(\mathbf{a}) \\
S_{i k}(\rho)
\end{array}\right)
$$

THEOREM 3.14. The vector field

$$
\boldsymbol{A}^{\text {scat }}=\nabla \times S_{k}[\mathbf{a}](\boldsymbol{x})-S_{k}[\boldsymbol{n} \rho](\boldsymbol{x})+i \eta\left(S_{k}\left[\boldsymbol{n} \times S_{i k} \mathbf{a}\right](\boldsymbol{x})+\nabla S_{k}\left[S_{i k} \rho\right](\boldsymbol{x})\right),
$$


with tangential density a of class $C_{t}(\partial D)$, scalar function $\rho$ of class $C(\partial D), \eta>0$, and constants $\left\{v_{j}\right\}_{j=1}^{N}$ solves the vector modified Dirichlet problem (with $k \geq 0$ ) provided that $\mathbf{a}, \rho$, and $\left\{v_{j}\right\}_{j=1}^{N}$ solve the equations

$$
\begin{aligned}
& \quad \frac{1}{2}\left(\begin{array}{l}
\mathbf{a} \\
\rho
\end{array}\right)+\overline{\bar{L}}\left(\begin{array}{l}
\mathbf{a} \\
\rho
\end{array}\right)+i \eta \overline{\bar{R}} \overline{\bar{S}}_{i k}\left(\begin{array}{l}
\mathbf{a} \\
\rho
\end{array}\right)+\left(\begin{array}{c}
0 \\
\sum_{j=1}^{N} v_{j} \chi_{j}
\end{array}\right)=\left(\begin{array}{l}
\mathbf{f} \\
h
\end{array}\right), \\
& \int_{\partial D_{j}}\left(-\boldsymbol{n} \cdot S_{k}(\boldsymbol{n} \rho)\right. \\
& \left.\quad+i \eta\left(\boldsymbol{n} \cdot S_{k}\left(\boldsymbol{n} \times S_{i k}(\mathbf{a})\right)-\frac{S_{i k} \rho}{2}+S_{k}^{\prime}\left(S_{i k} \rho\right)\right)\right) d s=q_{j},
\end{aligned}
$$

where $\chi_{j}$ denotes the characteristic function for boundary $\partial D_{j}$. The resulting integral equation is of the second kind on $C_{t}(\partial D) \times C(\partial D) \times \mathbb{C}^{N}$.

Proof. See Appendix B.

DEFINITION 3.15. We will refer to (3.44) and (3.51) as the scalar and vector decoupled potential integral equations. The former will be abbreviated by DPIEs and the latter by DPIEv. Together, they form the DPIE.

The following two theorems show that the solutions to the modified Dirichlet problems are continuous functions of the boundary data all the way to $k=0$. In particular, they are independent of the genus of $\partial D$. (As expected, the more regular the boundary data, the more regular the corresponding solution.)

THEOREM 3.16. The scalar modified Dirichlet problem has a unique solution for $k \geq 0$. Further, for continuous boundary data $f \in C(\partial D)$, the solution can be extended continuously up to the boundary. The following stability condition holds uniformly on the interval $\left[0, k_{\max }\right]$ :

$$
\left\|\phi^{\text {scat }}\right\|_{\infty, \mathbb{R}^{3} \backslash D} \leq K_{\left(\partial D, k_{\max }\right)}\left(\|f\|_{\infty, \partial D}+\sum_{j=1}^{N}\left|Q_{j}\right|^{2}\right) .
$$

For uniformly Hölder-continuous boundary data $f \in C^{0, \alpha}(\partial D)$, the solution can be extended in a uniformly Hölder-continuous way up to the boundary $\phi^{\text {scat }} \in$ $C^{0, \alpha}\left(\mathbb{R}^{3} \backslash D\right)$. The following stability condition holds uniformly on the interval $\left[0, k_{\max }\right]$ :

$$
\left\|\phi^{\mathrm{scat}}\right\|_{\alpha, \mathbb{R}^{3} \backslash D} \leq K_{\left(\alpha, \partial D, k_{\max }\right)}\left(\|f\|_{\alpha, \partial D}+\sum_{j=1}^{N}\left|Q_{j}\right|^{2}\right) .
$$

For uniformly Hölder-continuous differentiable boundary data $f \in C^{1, \alpha}(\partial D)$, the solution can be extended in a uniformly Hölder-continuous differentiable way 
up to the boundary $\phi^{\text {scat }} \in C^{1, \alpha}\left(\mathbb{R}^{3} \backslash D\right)$. The following stability condition holds uniformly on the interval $\left[0, k_{\max }\right]$ :

$$
\begin{aligned}
\left\|\phi^{\text {scat }}\right\|_{\alpha, \mathbb{R}^{3} \backslash D} \leq K_{\left(\alpha, \partial D, k_{\max }\right)}\left(\|f\|_{\alpha, \partial D}+\sum_{j=1}^{N}\left|Q_{j}\right|^{2}\right) \\
\left\|\nabla \phi^{\text {scat }}\right\|_{\alpha, \mathbb{R}^{3} \backslash D} \leq K_{\left(\alpha, \partial D, k_{\max }\right)}\left(\|f\|_{\alpha, \partial D}+\left\|\nabla_{s} f\right\|_{\alpha, \partial D}+\sum_{j=1}^{N}\left|Q_{j}\right|^{2}\right) .
\end{aligned}
$$

The constants $K$ depend only on the indicated indices.

Proof. See Appendix B.

THEOREM 3.17. The vector modified Dirichlet problem has a unique solution for $k \geq 0$. Further, for continuous boundary data $\mathbf{f} \in C_{t}(\partial D), h \in C(\partial D)$, the divergence and tangent components of the solution can be extended continuously up to the boundary. The following stability condition holds uniformly on the interval $\left[0, k_{\max }\right]$ :

$$
\left\|\boldsymbol{A}^{\mathrm{scat}}\right\|_{\infty, \mathfrak{M}} \leq K_{\left(\partial D, k_{\max }, \mathfrak{M}\right)}\left(\|\mathbf{f}\|_{\infty, \partial D}+\|h\|_{\infty, \partial D}+\sum_{j=1}^{N}\left|q_{j}\right|^{2}\right) ;
$$

for any closed $\mathfrak{M} \subset \mathbb{R}^{3} \backslash \bar{D}$

$$
\left\|\nabla \cdot \boldsymbol{A}^{\mathrm{scat}}\right\|_{\infty, \mathbb{R}^{3} \backslash D} \leq K_{\left(\partial D, k_{\max }\right)}\left(\|\mathbf{f}\|_{\infty, \partial D}+\|h\|_{\infty, \partial D}+\sum_{j=1}^{N}\left|q_{j}\right|^{2}\right) .
$$

For uniformly Hölder-continuous boundary data $\mathbf{f} \in C_{t}^{0, \alpha}(\partial D), h \in C^{0, \alpha}(\partial D)$, the solution and its divergence can be extended in a uniformly Hölder-continuous way up to the boundary $\boldsymbol{A} \in C^{0, \alpha}\left(\mathbb{R}^{3} \backslash D\right), \nabla \cdot \boldsymbol{A} \in C^{0, \alpha}\left(\mathbb{R}^{3} \backslash D\right)$. The following stability condition holds uniformly on the interval $\left[0, k_{\max }\right]$ :

$$
\begin{aligned}
\left\|\boldsymbol{A}^{\mathrm{scat}}\right\|_{\alpha, \mathbb{R}^{3} \backslash D} \leq K_{\left(\alpha, \partial D, k_{\max }\right)}\left(\|\mathbf{f}\|_{\alpha, \partial D}+\|h\|_{\alpha, \partial D}+\sum_{j=1}^{N}\left|q_{j}\right|^{2}\right), \\
\left\|\nabla \cdot \boldsymbol{A}^{\mathrm{scat}}\right\|_{\alpha, \mathbb{R}^{3} \backslash D} \leq K_{\left(\alpha, \partial D, k_{\max }\right)}\left(\|\mathbf{f}\|_{\alpha, \partial D}+\|h\|_{\alpha, \partial D}+\sum_{j=1}^{N}\left|q_{j}\right|^{2}\right) .
\end{aligned}
$$

For boundary data $\mathbf{f} \in C_{t}^{0, \alpha}(\operatorname{div}, \partial D), h \in C^{0, \alpha}(\partial D)$, the solution and its divergence and curl can be extended in a uniformly Hölder-continuous way up to the boundary $\boldsymbol{A} \in C^{0, \alpha}\left(\mathbb{R}^{3} \backslash D\right), \nabla \times \boldsymbol{A} \in C^{0, \alpha}\left(\mathbb{R}^{3} \backslash D\right), \nabla \cdot \boldsymbol{A} \in C^{0, \alpha}\left(\mathbb{R}^{3} \backslash D\right)$. 
The following stability condition holds uniformly on the interval $\left[0, k_{\max }\right]$ :

$$
\begin{gathered}
\left\|\boldsymbol{A}^{\text {scat }}\right\|_{\alpha, \mathbb{R}^{3} \backslash D} \leq K_{\left(\alpha, \partial D, k_{\max }\right)}\left(\|\mathbf{f}\|_{\alpha, \partial D}+\|h\|_{\alpha, \partial D}+\sum_{j=1}^{N}\left|q_{j}\right|^{2}\right), \\
\left\|\nabla \cdot \boldsymbol{A}^{\mathrm{scat}}\right\|_{\alpha, \mathbb{R}^{3} \backslash D} \leq K_{\left(\alpha, \partial D, k_{\max }\right)}\left(\|\mathbf{f}\|_{\alpha, \partial D}+\|h\|_{\alpha, \partial D}+\sum_{j=1}^{N}\left|q_{j}\right|^{2}\right), \\
\left\|\nabla \times \boldsymbol{A}^{\mathrm{scat}}\right\|_{\alpha, \mathbb{R}^{3} \backslash D} \leq \\
K_{\left(\alpha, \partial D, k_{\max }\right)}\left(\|\mathbf{f}\|_{\alpha, \partial D}+\left\|\nabla_{S} \cdot \mathbf{f}\right\|_{\alpha, \partial D}+\|h\|_{\alpha, \partial D}+\sum_{j=1}^{N}\left|q_{j}\right|^{2}\right)
\end{gathered}
$$

Proof. See Appendix B.

\section{Electromagnetic Scattering and Modified Dirichlet Problems}

In this section, we explain the connection between the scalar and vector modified Dirichlet problems and the Maxwell equations. It is evident from Theorems 3.16 and 3.17 that, if such a reformulation exists, then we have overcome the topological low-frequency breakdown that makes electromagnetic scattering from surfaces with nontrivial genus so difficult at low frequency.

We will first show that the vector and scalar modified Dirichlet problems preserve the Lorenz gauge, so that the induced $\boldsymbol{E}$ and $\boldsymbol{H}$ fields are Maxwellian. We will also show that the calculation is stable in the sense that bounded "incoming" data leads to bounded "outgoing" data, independent of the frequency. We will then show, in Theorem 4.3 , that the modified Dirichlet problems lead directly to the solution of the desired scattering problem.

Remark 4.1. For $\omega \in\left[0,+\infty\left[\right.\right.$ fixed, we consider the incoming fields $\boldsymbol{A}^{\text {inc }}, \phi^{\text {inc }}$, $\boldsymbol{E}^{\text {inc }}, \boldsymbol{H}^{\text {inc }}$ to be either suitably defined plane waves or induced by known current and charge distributions located in the exterior region. In either case, they are welldefined solutions to the homogeneous vector or scalar Helmholtz equation in some region that contains $\bar{D}$ :

$$
\begin{aligned}
\Delta \phi^{\mathrm{inc}}(\boldsymbol{x})+k^{2} \phi^{\mathrm{inc}}(\boldsymbol{x}) & =0, \\
\Delta \boldsymbol{A}^{\mathrm{inc}}(\boldsymbol{x})+k^{2} \boldsymbol{A}^{\mathrm{inc}}(\boldsymbol{x}) & =0, \quad \boldsymbol{x} \in \Omega, \\
\Delta \boldsymbol{E}^{\mathrm{inc}}(\boldsymbol{x})+k^{2} \boldsymbol{E}^{\mathrm{inc}}(\boldsymbol{x}) & =0, \quad \\
\Delta \boldsymbol{H}^{\mathrm{inc}}(\boldsymbol{x})+k^{2} \boldsymbol{H}^{\mathrm{inc}}(\boldsymbol{x}) & =0,
\end{aligned}
$$

with

$$
\phi^{\text {inc }}, \boldsymbol{A}^{\text {inc }}, \boldsymbol{E}^{\text {inc }}, \boldsymbol{H}^{\text {inc }} \in C^{2}(\Omega) \cap C^{0, \alpha}(\bar{\Omega}),
$$


where $\Omega$ is an open set that contains $\bar{D}$. We write $\phi_{\omega}^{\mathrm{inc}}, \boldsymbol{A}_{\omega}^{\mathrm{inc}}, \boldsymbol{E}_{\omega}^{\mathrm{inc}}, \boldsymbol{H}_{\omega}^{\mathrm{inc}}$ to denote explicitly the dependence on frequency $\omega$. We assume that the maps $\omega \rightarrow$ $\phi_{\omega}^{\text {inc }}, \boldsymbol{A}_{\omega}^{\mathrm{inc}}, \boldsymbol{E}_{\omega}^{\mathrm{inc}}, \boldsymbol{H}_{\omega}^{\mathrm{inc}}$ are continuous functions defined from $\left[0,+\infty\left[\right.\right.$ to $C^{0, \alpha}(\bar{\Omega})$.

THEOREM 4.2. Let $\boldsymbol{A}^{\mathrm{inc}}, \phi^{\text {inc }}$ be bounded (for $\omega \rightarrow 0$ ) incoming vector and scalar Helmholtz potentials in the Lorenz gauge:

$$
\nabla \cdot \mathbf{A}^{\mathrm{inc}}(\mathbf{x})=i \omega \mu \epsilon \phi^{\mathrm{inc}}(\mathbf{x})
$$

for $(\mathbf{x}) \in \Omega$. Then, the associated vector and scalar scattered Helmholtz potentials $\boldsymbol{A}^{\text {scat }}, \phi^{\text {scat }}$ (see Definition 3.5 are also bounded and satisfy the Lorenz gauge condition

$$
\nabla \cdot \mathbf{A}^{\text {scat }}(\mathbf{x})=i \omega \mu \epsilon \phi^{\text {scat }}(\mathbf{x})
$$

for $\mathbf{x} \in \mathbb{R}^{3} \backslash D$.

Proof. Due to the analyticity of $\boldsymbol{A}^{\text {inc }}$ and $\phi^{\text {inc }}$, the boundary data is smooth. This implies that $\phi^{\text {scat }} \in C^{1, \alpha}\left(\mathbb{R}^{3} \backslash D\right), A^{\text {scat }} \in C^{2}\left(\mathbb{R}^{3} \backslash \bar{D}\right) \cap C^{0, \alpha}\left(\mathbb{R}^{3} \backslash D\right)$, $\nabla \cdot \boldsymbol{A}^{\text {scat }} \in C^{0, \alpha}\left(\mathbb{R}^{3} \backslash D\right)$, and $\nabla \times \boldsymbol{A}^{\text {scat }} \in C^{0, \alpha}\left(\mathbb{R}^{3} \backslash D\right)$ (see Theorems 3.16 and 3.17). This implies that we can evaluate the flux conditions on the surfaces $\partial D_{j}$ without using the parallel surface trick $\partial D_{j}^{+}$.

By Lemma 3.6, the scalar Helmholtz potential $\psi^{\text {scat }}=\nabla \cdot \boldsymbol{A}^{\text {scat }}$ satisfies

$$
\begin{gathered}
\psi^{\text {scat }}=h+v_{j}=-\nabla \cdot \boldsymbol{A}^{\mathrm{inc}}+\left.v_{j}\right|_{\partial D}, \\
\int_{\partial D_{j}} \frac{\partial \psi^{\text {scat }}}{\partial n} d s=-k^{2} q_{j}=k^{2} \int_{\partial D_{j}} \mathbf{n} \cdot \mathbf{A}^{\mathrm{inc}} d s .
\end{gathered}
$$

Using the Lorenz gauge condition on the boundary itself, we may write

$$
\psi^{\text {scat }}=h+v_{j}=-i \omega \mu \epsilon \phi^{\text {inc }}+\left.v_{j}\right|_{\partial D} .
$$

Since

$$
\nabla \cdot\left(i \omega A^{\mathrm{inc}}-\nabla \phi^{\mathrm{inc}}\right)=0, \quad \boldsymbol{x} \in \Omega,
$$

we have

$$
\int_{\partial D_{j}} \frac{\partial \phi^{\mathrm{inc}}}{\partial n} d s=i \omega \int_{\partial D_{j}} \mathbf{n} \cdot \mathbf{A}^{\mathrm{inc}} d s .
$$

Thus,

$$
\begin{aligned}
\int_{\partial D_{j}} \frac{\partial \psi^{\mathrm{scat}}}{\partial n} d s=k^{2} \int_{\partial D_{j}} \mathbf{n} \cdot \mathbf{A}^{\mathrm{inc}} d s & =\frac{k^{2}}{i \omega} \int_{\partial D_{j}} \frac{\partial \phi^{\mathrm{inc}}}{\partial n} d s \\
& =-i \omega \mu \epsilon \int_{\partial D_{j}} \frac{\partial \phi^{\mathrm{inc}}}{\partial n} d s .
\end{aligned}
$$


F. VICO ET AL.

From (4.5) and (4.8), we see that the function and constants $\psi^{\text {scat }},\left\{v_{j}\right\}_{j=1}^{N}$ and the function and constants $i \omega \mu \epsilon \phi^{\text {scat }},\left\{i \omega \mu \epsilon v_{j}\right\}_{j=1}^{N}$ satisfy the same scalar modified Dirichlet problem. By uniqueness (Theorem 3.9), we find that

$$
i \omega \mu \epsilon \phi^{\text {scat }}(\mathbf{x})=\psi^{\text {scat }}(\mathbf{x})=\nabla \cdot A^{\text {scat }}(\mathbf{x}), \quad \mathbf{x} \in \mathbb{R}^{3} \backslash D,
$$

so that $\boldsymbol{A}^{\text {scat }}$ and $\phi^{\text {scat }}$ are in the Lorenz gauge.

Using the estimates 3.53 and 3.57 of Theorems 3.16 and 3.17 , we find that $\boldsymbol{A}^{\text {scat }}, \phi^{\text {scat }}$ are continuous functions of $\boldsymbol{A}^{\text {inc }}, \phi^{\text {inc }}$, uniformly on $\omega \in\left[0, \omega_{\max }\right]$ for any $\omega_{\max }$. In particular, they are uniformly continuous functions of $\mathbf{f}=-\boldsymbol{n} \times$ $\left.\boldsymbol{A}^{\text {inc }}\right|_{\partial D}, h=-\left.\nabla \cdot \boldsymbol{A}^{\text {inc }}\right|_{\partial D}=\left.i \omega \mu \epsilon \phi^{\text {inc }}\right|_{\partial D}, f=-\left.\phi^{\text {inc }}\right|_{\partial D}, q_{j}=-\int_{\partial D_{j}} \boldsymbol{n}$. $\boldsymbol{A}^{\text {inc }} d s$, and $Q_{j}=-\int_{\partial D_{j}} \frac{\partial \phi^{\text {inc }}}{\partial n} d s=-i \omega \int_{\partial D_{j}} \boldsymbol{n} \cdot \boldsymbol{A}^{\text {inc }}$. Since $\boldsymbol{A}^{\text {inc }}, \phi^{\text {inc }}$ are bounded in $C^{0, \alpha}(\bar{\Omega})$ (for $\omega \rightarrow 0$ ), $A^{\text {scat }}, \phi^{\text {scat }}$ are bounded in $C^{0, \alpha}\left(\mathbb{R}^{3} \backslash D\right)$.

The next theorem is the main result of the present paper.

THEOREM 4.3. For any $\omega \geq 0$, let $\mathbf{E}^{\mathrm{inc}}, \mathbf{H}^{\mathrm{inc}}$ be an incoming electromagnetic field described by the potentials $\boldsymbol{A}^{\text {inc }}, \phi^{\text {inc }}$ in the Lorenz gauge:

$$
\begin{gathered}
\boldsymbol{H}^{\mathrm{inc}}=\frac{1}{\mu} \nabla \times \boldsymbol{A}^{\mathrm{inc}}, \quad \boldsymbol{E}^{\mathrm{inc}}=i \omega \boldsymbol{A}^{\mathrm{inc}}-\nabla \phi^{\mathrm{inc}}, \\
\nabla \cdot \boldsymbol{A}^{\mathrm{inc}}=i \omega \mu \epsilon \phi^{\mathrm{inc}},
\end{gathered}
$$

and let $\boldsymbol{A}^{\text {scat }}, \phi^{\text {scat }}$ denote the corresponding scattered vector and scalar potentials (Definition 3.5). Then the electromagnetic fields $\mathbf{E}^{\text {scat }}, \mathbf{H}^{\text {scat }}$ scattered from a perfect conductor are given by

$$
\boldsymbol{H}^{\mathrm{scat}}=\frac{1}{\mu} \nabla \times \boldsymbol{A}^{\mathrm{scat}}, \quad \boldsymbol{E}^{\mathrm{scat}}=i \omega \boldsymbol{A}^{\mathrm{scat}}-\nabla \phi^{\mathrm{scat}} .
$$

with

$$
\begin{gathered}
\nabla \cdot \boldsymbol{A}^{\mathrm{scat}}=i \omega \mu \epsilon \phi^{\mathrm{scat}}, \\
\boldsymbol{n} \times \boldsymbol{E}^{\mathrm{scat}}=-\boldsymbol{n} \times\left.\boldsymbol{E}^{\mathrm{inc}}\right|_{\partial D}, \quad \boldsymbol{n} \cdot \boldsymbol{H}^{\mathrm{scat}}=-\left.\boldsymbol{n} \cdot \boldsymbol{H}^{\mathrm{inc}}\right|_{\partial D} \cdot
\end{gathered}
$$

When the explicit dependence on $\omega$ is needed, we write $\boldsymbol{E}_{\omega}^{\mathrm{scat}}$ and $\boldsymbol{H}_{\omega}^{\text {scat }}$ instead of $\boldsymbol{E}^{\text {scat }}$ and $\boldsymbol{H}^{\text {scat }}$. With this notation, the scattered electromagnetic fields at zero frequency are the low-frequency limits of the time-harmonic solutions. That is,

$$
\begin{gathered}
\lim _{\omega \rightarrow 0}\left\|\boldsymbol{E}_{\omega}^{\text {scat }}-\boldsymbol{E}_{0}^{\text {scat }}\right\|_{\alpha, \mathbb{R}^{3} / D} \rightarrow 0, \\
\lim _{\omega \rightarrow 0}\left\|\boldsymbol{H}_{\omega}^{\text {scat }}-\boldsymbol{H}_{0}^{\text {scat }}\right\|_{\alpha, \mathbb{R}^{3} / D} \rightarrow 0 .
\end{gathered}
$$

PROOF. As in the previous theorem, due to the analyticity of $\boldsymbol{A}^{\text {inc }}$ and $\phi^{\text {inc }}$, the boundary data is smooth. Therefore $\phi^{\text {scat }} \in C^{1, \alpha}\left(\mathbb{R}^{3} \backslash D\right), A^{\text {scat }} \in C^{2}\left(\mathbb{R}^{3} \backslash \bar{D}\right) \cap$ $C^{0, \alpha}\left(\mathbb{R}^{3} \backslash D\right), \nabla \cdot \boldsymbol{A}^{\text {scat }} \in C^{0, \alpha}\left(\mathbb{R}^{3} \backslash D\right)$, and $\nabla \times \boldsymbol{A}^{\text {scat }} \in C^{0, \alpha}\left(\mathbb{R}^{3} \backslash D\right.$ ) (see Theorems 3.16 and 3.17). 
Since $\boldsymbol{A}^{\text {scat }}, \phi^{\text {scat }}$ are Helmholtz potentials in the Lorenz gauge, the associated $\boldsymbol{E}^{\text {scat }}, \boldsymbol{H}^{\text {scat }}$ are valid Maxwell fields that satisfy the necessary radiation condition. We need only check that the desired boundary conditions are satisfied. From the boundary conditions on $\boldsymbol{A}^{\text {scat }}, \phi^{\text {scat }}$ we have

$$
\begin{array}{cl}
\boldsymbol{n} \times \boldsymbol{A}^{\text {scat }}=-\boldsymbol{n} \times\left.\boldsymbol{A}^{\mathrm{inc}}\right|_{\partial D}, & i \omega \boldsymbol{n} \times \boldsymbol{A}^{\text {scat }}=-i \omega \boldsymbol{n} \times\left.\boldsymbol{A}^{\mathrm{inc}}\right|_{\partial D}, \\
\phi^{\text {scat }}=-\phi^{\text {inc }}+\left.V_{j}\right|_{\partial D_{j}}, & \boldsymbol{n} \times \nabla \phi^{\text {scat }}=-\boldsymbol{n} \times\left.\nabla \phi^{\mathrm{inc}}\right|_{\partial D .}
\end{array}
$$

Adding (4.11) and (4.12), we have

$$
\begin{aligned}
i \omega \boldsymbol{n} \times \boldsymbol{A}^{\text {scat }}-\boldsymbol{n} \times \nabla \phi^{\text {scat }} & =-i \omega \boldsymbol{n} \times \boldsymbol{A}^{\mathrm{inc}}+\boldsymbol{n} \times\left.\nabla \phi^{\mathrm{inc}}\right|_{\partial D}, \\
\boldsymbol{n} \times \boldsymbol{E}^{\text {scat }} & =-\boldsymbol{n} \times\left.\boldsymbol{E}^{\text {inc }}\right|_{\partial D} .
\end{aligned}
$$

Taking the surface divergence of (4.11), we also have that

$$
\begin{gathered}
\boldsymbol{n} \times \boldsymbol{A}^{\text {scat }}=-\boldsymbol{n} \times\left.\boldsymbol{A}^{\mathrm{inc}}\right|_{\partial D}, \quad \nabla_{S} \cdot \boldsymbol{n} \times \boldsymbol{A}^{\text {scat }}=-\nabla_{S} \cdot \boldsymbol{n} \times\left.\boldsymbol{A}^{\mathrm{inc}}\right|_{\partial D}, \\
\boldsymbol{n} \cdot \boldsymbol{H}^{\text {scat }}=-\left.\boldsymbol{n} \cdot \boldsymbol{H}^{\text {inc }}\right|_{\partial D} .
\end{gathered}
$$

Thus, for $\omega>0$ we have the correct solution for $\boldsymbol{E}_{\omega}^{\text {scat }}$ and $\boldsymbol{H}_{\omega}^{\text {scat }}$.

We now prove that our particular solution to the static Maxwell equations, $\boldsymbol{E}_{0}^{\text {scat }}$ and $\boldsymbol{H}_{0}^{\text {scat }}$, corresponds precisely to the limiting case of the time-harmonic solution as $\omega \rightarrow 0$. That is,

(4.15) $\lim _{\omega \rightarrow 0}\left\|\boldsymbol{E}_{\omega}^{\text {scat }}-\boldsymbol{E}_{0}^{\text {scat }}\right\|_{\alpha, \mathbb{R}^{3} / D} \rightarrow 0, \quad \lim _{\omega \rightarrow 0}\left\|\boldsymbol{H}_{\omega}^{\text {scat }}-\boldsymbol{H}_{0}^{\text {scat }}\right\|_{\alpha, \mathbb{R}^{3} / D} \rightarrow 0$.

For this, we will require the following equalities:

$$
\begin{gathered}
\lim _{\omega \rightarrow 0}\left\|-\boldsymbol{n} \times \boldsymbol{E}_{\omega}^{\mathrm{inc}}+\boldsymbol{n} \times \boldsymbol{E}_{0}^{\mathrm{inc}}\right\|_{\alpha, \partial D}=0, \\
\lim _{\omega \rightarrow 0}\left\|\frac{1}{i \omega \mu} \nabla_{s} \cdot-\boldsymbol{n} \times \boldsymbol{E}_{\omega}^{\mathrm{inc}}+\boldsymbol{n} \cdot \boldsymbol{H}_{0}^{\mathrm{inc}}\right\|_{\alpha, \partial D}=0, \\
\lim _{\omega \rightarrow 0} \frac{1}{-i \omega \mu} \int_{\partial D} \boldsymbol{n} \times \boldsymbol{E}_{\omega}^{\mathrm{inc}} \cdot \mathbf{Z}_{\mathbf{m}} d s=\int_{\mathbb{R}^{3} / D} \boldsymbol{H}_{0}^{\mathrm{scat}} \cdot \mathbf{Z}_{\mathbf{m}} d v, \\
\int_{\mathbb{R}^{3 / D}} \boldsymbol{E}_{0}^{\mathrm{scat}} \cdot \mathbf{Y}_{\mathbf{j}} d v=0 .
\end{gathered}
$$

Condition 4.16 follows from the assumption that $\lim _{\omega \rightarrow 0}\left\|\boldsymbol{E}_{\omega}^{\mathrm{inc}}-\boldsymbol{E}_{0}^{\mathrm{inc}}\right\|_{\alpha, \bar{\Omega}}=0$. From the fact that $\nabla_{s} \cdot-\boldsymbol{n} \times \boldsymbol{E}_{\omega}^{\mathrm{inc}}=i \omega \mu \boldsymbol{n} \cdot \boldsymbol{H}_{\omega}^{\text {inc }}$, we obtain 4.17. Note now that

$$
\begin{aligned}
\frac{1}{-i \omega \mu} \int_{\partial D} \boldsymbol{n} \times \boldsymbol{E}_{\omega}^{\mathrm{inc}} \cdot \mathbf{Z}_{\mathbf{m}} d s & =\frac{1}{-i \omega \mu} \int_{\partial D} \boldsymbol{n} \times\left(i \omega \boldsymbol{A}_{\omega}^{\mathrm{inc}}-\nabla \phi_{\omega}^{\mathrm{inc}}\right) \cdot \mathbf{Z}_{\mathbf{m}} d s \\
& =\frac{-1}{\mu} \int_{\partial D} \boldsymbol{n} \times \boldsymbol{A}_{\omega}^{\mathrm{inc}} \cdot \mathbf{Z}_{\mathbf{m}} d s,
\end{aligned}
$$


where $\left\{\mathbf{Z}_{\mathbf{m}}\right\}_{m=1}^{g}$ is a basis for the Neumann fields in the exterior region. Gauss' theorem then yields

$$
\begin{aligned}
\int_{\partial D}-\boldsymbol{n} \times \nabla \phi^{\text {inc }} \cdot \mathbf{Z}_{\mathbf{m}} d s & =-\int_{\partial D} \nabla_{s} \cdot \boldsymbol{n} \times \mathbf{Z}_{\mathbf{m}} \phi^{\text {inc }} d s \\
& =\int_{\partial D} \boldsymbol{n} \cdot \nabla \times \mathbf{Z}_{\mathbf{m}} \phi^{\text {inc }} d s=0 .
\end{aligned}
$$

Thus,

$$
\lim _{\omega \rightarrow 0} \frac{1}{-i \omega \mu} \int_{\partial D} \boldsymbol{n} \times \boldsymbol{E}_{\omega}^{\mathrm{inc}} \cdot \mathbf{Z}_{\mathbf{m}} d s=\frac{-1}{\mu} \int_{\partial D} \boldsymbol{n} \times \boldsymbol{A}_{0}^{\mathrm{inc}} \cdot \mathbf{Z}_{\mathbf{m}} d s
$$

and

$$
\begin{aligned}
\int_{\mathbb{R}^{3} / D} \boldsymbol{H}_{0}^{\text {scat }} \cdot \mathbf{Z}_{\mathbf{m}} d v & =\int_{\mathbb{R}^{3} / D} \frac{1}{\mu} \nabla \times \boldsymbol{A}_{0}^{\text {scat }} \cdot \nabla \times \mathbf{A}_{\mathbf{m}} d v \\
& =\frac{1}{\mu} \int_{\partial D} \boldsymbol{n} \times \boldsymbol{A}_{0}^{\text {scat }} \cdot \mathbf{Z}_{\mathbf{m}} d s \\
& =\frac{-1}{\mu} \int_{\partial D} \boldsymbol{n} \times \boldsymbol{A}_{0}^{\text {inc }} \cdot \mathbf{Z}_{\mathbf{m}} d s
\end{aligned}
$$

where $\mathbf{A}_{\mathbf{m}}$ is defined in (5.28) of [10] with the properties $\nabla \times \mathbf{A}_{\mathbf{m}}=\mathbf{Z}_{\mathbf{m}}$ and $\nabla \cdot \mathbf{A}_{\mathbf{m}}=0$. The previous result is obtained from Green's theorem, taking into account the radiation condition for static fields and $\nabla \cdot \mathbf{A}_{\mathbf{m}}=0$. From (4.22) and (4.23) we get 4.18). Condition 4.19) follows from the calculation

$$
\begin{aligned}
\int_{\mathbb{R}^{3} / D} \boldsymbol{E}_{0}^{\text {scat }} \cdot \mathbf{Y}_{\mathbf{j}} d v & =-\int_{\mathbb{R}^{3} / D} \nabla \phi_{0}^{\text {scat }} \cdot \nabla u_{j} d v \\
& =-\int_{\partial D_{j}} \frac{\partial \phi_{0}^{\text {scat }}}{\partial n} d s=\int_{\partial D_{j}} \frac{\partial \phi_{0}^{\text {inc }}}{\partial n} d s=\int_{D} \Delta \phi_{0}^{\text {inc }} d v=0,
\end{aligned}
$$

where $u_{j}$ is a harmonic scalar field in the exterior region with the property $\left.u_{j}\right|_{\partial D}=$ $\mathbb{1}_{\partial D_{j}}$ and $\mathbf{Y}_{\mathbf{j}}=\nabla u_{j}$ is a basis for the Dirichlet fields.

The desired result (4.10) now follows from theorem 5.11 in [10] (which describes technical conditions for convergence of Maxwell fields in the low-frequency limit.)

The consistency conditions (1.19) on the flux of the magnetic field through each hole $S_{j}[14]$ are also easily verified for all $\omega \geq 0$ :

$$
\boldsymbol{n} \times \boldsymbol{A}^{\mathrm{scat}}=-\boldsymbol{n} \times\left.\boldsymbol{A}^{\mathrm{inc}}\right|_{\partial D}, \quad \oint_{\boldsymbol{B}_{j}} \boldsymbol{A}^{\mathrm{scat}} \cdot d \mathbf{l}=-\oint_{\boldsymbol{B}_{j}} \boldsymbol{A}^{\mathrm{inc}} \cdot d \mathbf{l} .
$$


Here, $B_{j}$ is a standard $B$-cycle on a surface with nonzero genus (see Figure 1.1 and related discussion).

\section{Incoming Potentials}

In a stable DPIE approach, the vector and scalar potentials must be defined in the Lorenz gauge and be bounded as $\omega \rightarrow 0$. We will need to find a representation for the incoming fields that will permit the stable uncoupling of the vector and scalar potentials. Assuming we are given the "impressed" free current and charge $\boldsymbol{J}^{\text {imp }}, \rho^{\text {imp }}$, the incoming potentials

$$
\boldsymbol{A}^{\mathrm{inc}}(\boldsymbol{x})=\mu S_{k}\left[\boldsymbol{J}^{\mathrm{imp}}\right](\boldsymbol{x}), \quad \phi^{\mathrm{inc}}(\boldsymbol{x})=\frac{1}{\epsilon} S_{k}\left[\rho^{\mathrm{imp}}\right](\boldsymbol{x}),
$$

satisfy these requirements. For an incoming plane wave with a polarization vector $\boldsymbol{E}_{p}$ and a direction of propagation $\mathbf{u}$, given by

$$
\boldsymbol{E}^{\mathrm{inc}}=\boldsymbol{E}_{p} e^{i k \mathbf{u} \cdot \mathbf{x}}, \quad \boldsymbol{H}^{\mathrm{inc}}=\boldsymbol{H}_{p} e^{i k \mathbf{u} \cdot \mathbf{x}}=\frac{\mathbf{u} \times \boldsymbol{E}_{p}}{Z} e^{i k \mathbf{u} \cdot \mathbf{x}},
$$

where $Z=\sqrt{\mu / \epsilon}$, the standard representation of incoming vector and scalar potentials

$$
\boldsymbol{A}^{\mathrm{inc}}=\frac{1}{i \omega} \boldsymbol{E}^{\mathrm{inc}}, \quad \phi^{\mathrm{inc}}=0,
$$

does not lead to stable uncoupling, since the vector potential is unbounded as $\omega \rightarrow 0$. But, as mentioned above, the Lorenz gauge does not, by itself, impose uniqueness on the governing potentials. It is easy to check that the vector and scalar potentials defined by

$$
\boldsymbol{A}^{\prime \text { inc }}=-\mathbf{u}\left(\boldsymbol{x} \cdot \boldsymbol{E}_{p}\right) \sqrt{\mu \epsilon} e^{i k \mathbf{u} \cdot \boldsymbol{x}}, \quad \phi^{\prime \text { inc }}=-\boldsymbol{x} \cdot \boldsymbol{E}_{p} e^{i k \mathbf{u} \cdot \boldsymbol{x}},
$$

satisfy the Lorenz gauge condition

$$
\nabla \cdot \boldsymbol{A}^{\prime \text { inc }}(\boldsymbol{x})=i \omega \mu \epsilon \phi^{\prime \text { inc }}(\boldsymbol{x})
$$

both $\boldsymbol{A}^{\prime \text { inc }}$ and $\phi^{\prime \text { inc }}$ are bounded Helmholtz potentials as $\omega \rightarrow 0$ and represent the same incoming plane wave (5.2). See Appendix C for more details on how to stably decompose incoming/outgoing electric and magnetic multipole fields (Debye sources).

\section{The DPIE and the Aharonov-Bohm Effect}

In classical physics, the Maxwell equations are described in terms of the components of the electric and magnetic fields, with the vector and scalar potentials viewed as matters of computational convenience. In quantum mechanics, however, it was shown by Aharonov and Bohm [2] that an electron is sensitive to the vector potential $\boldsymbol{A}$ itself in regions where $\boldsymbol{E}$ and $\boldsymbol{H}$ are identically zero (the AharonovBohm effect). 


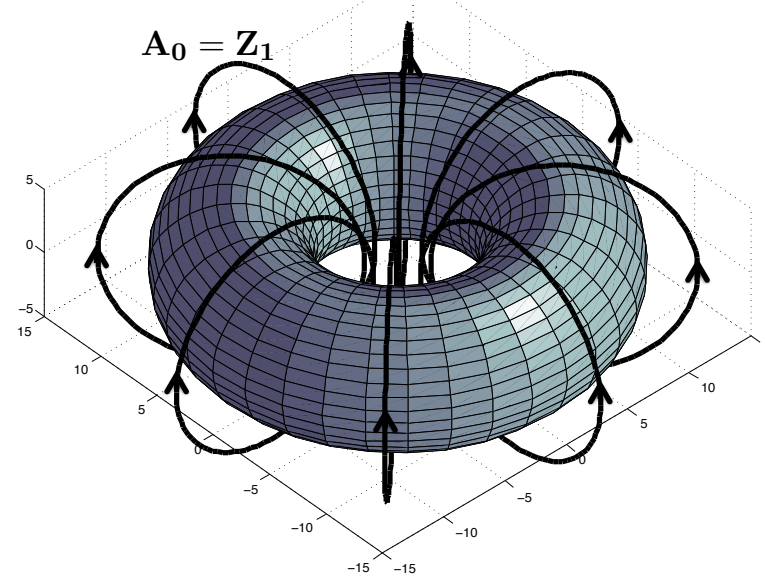

FigURE 6.1. In the exterior of a torus, a harmonic Neumann field $Z_{1}$ serves as a vector potential $\boldsymbol{A}_{0}$ with the corresponding scalar potential set equal to zero. For $\boldsymbol{x} \in \mathbb{R}^{3} \backslash D$, the associated electromagnetic fields $\boldsymbol{E}$ and $\boldsymbol{H}$ are identically zero.

Let us first recall that the two pairs of potentials $\{\boldsymbol{A}, \phi\}$ and $\left\{\boldsymbol{A}^{\prime}, \phi^{\prime}\right\}$ produce the same electromagnetic field so long as they satisfy the condition

$$
\mathbf{A}^{\prime}=\mathbf{A}+\nabla \psi, \quad \phi^{\prime}=\phi+i \omega \psi .
$$

In multiply connected regions at zero frequency, however, the situation is more complex. There exist potentials that give rise to identical fields that are not related according to 6.1). In particular, the potentials

$$
\begin{array}{ll}
\boldsymbol{A}_{0}=\mathbf{Z}_{1}, & \phi_{0}=0, \\
\boldsymbol{A}_{0}^{\prime}=\mathbf{0}, & \phi_{0}^{\prime}=0,
\end{array}
$$

where $\mathbf{Z}_{\mathbf{1}}$ is an exterior harmonic Neumann field, give rise to zero electromagnetic fields in the exterior. $\mathbf{Z}_{\mathbf{1}}$, however, is not the gradient of a single-valued harmonic function.

The Aharonov-Bohm effect is based on an experiment that is able to distinguish between the physical states $\boldsymbol{A}_{0}, \phi_{0}$ and $\boldsymbol{A}_{0}^{\prime}, \phi_{0}^{\prime}$. We have taken some liberties with the actual experiment in [2], but the physical idea is the same. In essence, quantum mechanical tunneling permits an electron to be aware of the electromagnetic field in the interior of $D$ even though it is a perfect conductor. For $\boldsymbol{A}_{0}^{\prime}, \phi_{0}^{\prime}$, the field is identically zero in the interior, but for $\boldsymbol{A}_{0}, \phi_{0}$ it is not. As discussed in [10,13, $15,32], A_{0}$ can be viewed as the field induced by an axisymmetric current density flowing on the surface in the direction of the arrows in Figure 6.1. This induces a nontrivial magnetic field within the torus. Electrons, as a result, sense whether they traveled through the hole of the torus or passed by the torus on the outside. The DPIE formalism easily distinguishes between these two cases, since we deal with 
the vector and scalar potentials directly. Thus, $\boldsymbol{A}_{0}, \phi_{0}, \boldsymbol{A}_{0}^{\prime}$, and $\phi_{0}^{\prime}$ in 6.2 satisfy

$$
\begin{array}{lll}
\boldsymbol{n} \times\left.\boldsymbol{A}_{0}\right|_{\partial D}=\boldsymbol{n} \times \mathbf{Z}_{1}, & \left.\nabla \cdot \boldsymbol{A}_{0}\right|_{\partial D}=0, & \left.\phi_{0}\right|_{\partial D}=0, \\
\boldsymbol{n} \times\left.\boldsymbol{A}_{0}^{\prime}\right|_{\partial D}=\mathbf{0}, & \left.\nabla \cdot \boldsymbol{A}_{0}^{\prime}\right|_{\partial D}=0, & \left.\phi_{0}^{\prime}\right|_{\partial D}=0 .
\end{array}
$$

\section{The DPIE in the High-Frequency Regime}

Theorems 3.13 and 3.14 suggest that the numerical solution of scattering problems in the presence of perfect conductors can be effectively solved through the use of the DPIE, defined by equations (3.44) and (3.51). Both equations are Fredholm equations of the second kind, invertible for all frequencies. Our interest in the DPIE formulation grew out of issues in low-frequency scattering. Nevertheless, we would like to find a representation that is effective at all frequencies, and this will involve a slight rescaling of the equations. In order to carry out a suitable analysis, we follow [21] and study scattering from the unit sphere $\partial D=\{\boldsymbol{x}:\|\boldsymbol{x}\|=1\}$. For $k \leq 1$, setting $\eta=1$ works well, while for $k>1$ the optimal scaling factor $\eta \approx k$ (see [21]). Setting $\eta=k$, instead of (3.44), we have the scaled DPIEs integral equation:

$$
\begin{gathered}
\frac{\sigma}{2}+D_{k} \sigma-i k S_{k} \sigma-\sum_{j=1}^{N} V_{j} \chi_{j}=f, \\
\int_{\partial D_{j}}\left(\frac{1}{k}\left(D_{k}^{\prime}-D_{0}^{\prime}\right) \sigma+i \frac{\sigma}{2}-i S_{k}^{\prime} \sigma\right) d s=\frac{1}{k} Q_{j},
\end{gathered}
$$

where the second set of equations has been multiplied by a factor of $\frac{1}{k}$. For the vector modified Dirichlet problem, when $k>1$, we replace 3.50) with

$$
\begin{aligned}
\boldsymbol{A}^{\text {scat }}= & \nabla \times S_{k}[\mathbf{a}](\boldsymbol{x})-k S_{k}[\boldsymbol{n} \varrho](\boldsymbol{x}) \\
& +i k\left(k S_{k}\left[\boldsymbol{n} \times S_{i k} \mathbf{a}\right](\boldsymbol{x})+\nabla S_{k}\left[S_{i k} \varrho\right](\boldsymbol{x})\right),
\end{aligned}
$$

where we have multiplied the single-layer potential terms by $k$ and set $\eta=k$. We also rescale the boundary condition $\nabla \cdot \boldsymbol{A}^{\text {scat }}=-\nabla \cdot \boldsymbol{A}^{\text {scat }}+v_{n}$ in the modified Dirichlet problem, dividing each side by $k$. These changes lead to the scaled DPIEv integral equation:

$$
\begin{aligned}
& \frac{1}{2}\left(\begin{array}{l}
\mathbf{a} \\
\rho
\end{array}\right)+\overline{\bar{L}}_{s}\left(\begin{array}{l}
\mathbf{a} \\
\rho
\end{array}\right)+i k \overline{\bar{R}}_{s} \overline{\bar{S}}_{i k}\left(\begin{array}{l}
\mathbf{a} \\
\rho
\end{array}\right)+\left(\begin{array}{c}
0 \\
\sum_{j=1}^{N} v_{j} \chi_{j}
\end{array}\right)=\left(\begin{array}{c}
\mathbf{f} \\
\frac{1}{k} h
\end{array}\right), \\
& \int_{\partial D_{j}}\left(-k \boldsymbol{n} \cdot S_{k}(\boldsymbol{n} \varrho)\right. \\
& \left.\quad+i k\left(k \boldsymbol{n} \cdot S_{k}\left(\boldsymbol{n} \times S_{i k}(\mathbf{a})\right)-\frac{S_{i k} \varrho}{2}+S_{k}^{\prime}\left(S_{i k}(\varrho)\right)\right)\right) d s=q_{j},
\end{aligned}
$$




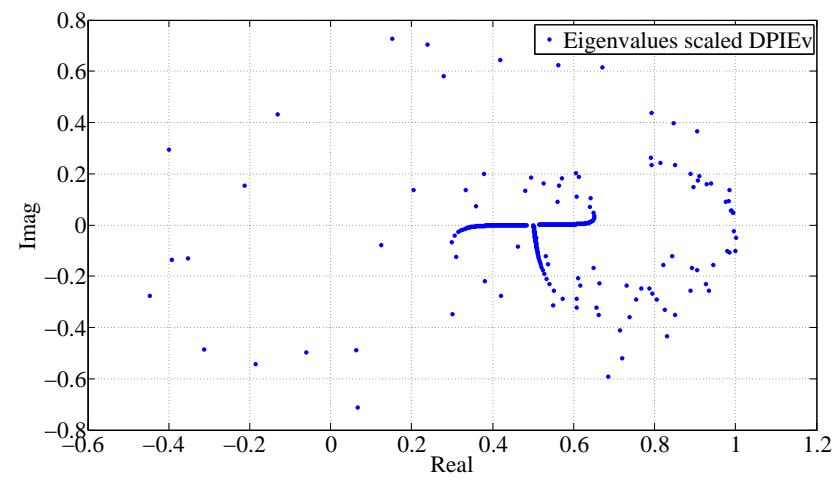

FIGURE 7.1. Spectrum of the scaled DPIEv integral equation (7.3) for a spherical scatterer of radius 1 at $k=30$. Note that there is one cluster point at $\lambda=0.5$.

where

$$
\overline{\overline{L_{s}}}=\left(\begin{array}{cc}
L_{11} & k L_{12} \\
\frac{1}{k} L_{21} & L_{22}
\end{array}\right), \quad \overline{\bar{R}}_{s}=\left(\begin{array}{cc}
k R_{11} & R_{12} \\
R_{21} & \frac{1}{k} R_{22}
\end{array}\right),
$$

with $L_{i j}, R_{i j}$ defined in (3.46) and 3.48 .

We turn now to the analysis of the DPIE on the unit sphere, where exact expressions for the various integral operators have been worked out in detail [31]. More precisely, by using scalar and vector spherical harmonics, each integral operator has a simple signature, which has been tabulated in [31]. This permits us to compute the condition number and spectrum of the DPIEs and DPIEv integral equations. In Figures 7.1 and 7.2 , we plot the spectrum and the singular values of the scaled DPIEv (7.3).

In Figure 7.3, we compare the condition numbers of the DPIEv and the scaled DPIEv. Equally revealing is to plot the spectrum of the DPIEv and the scaled DPIEv (Figure 7.4).

While the analysis above has been carried out only for a spherical scatterer, it is reasonable to expect that the scaled DPIEv is likely to have a big impact even for surfaces of arbitrary shape. As a final point of comparison, Figure 7.5 plots the condition number of various integral equations that have been suggested for the solution of the Maxwell equations over a wide range of frequencies. Note that the scaled DPIE produces slightly worse condition numbers for $1 \ll k$. We suspect that improvements in the DPIE representation may lead to better performance, but leave that for future research. Note, however, that the scaled DPIE is surprisingly effective in the high-frequency regime, despite the fact that it was conceived in order to overcome topological low-frequency breakdown for scatterers of nonzero genus. 


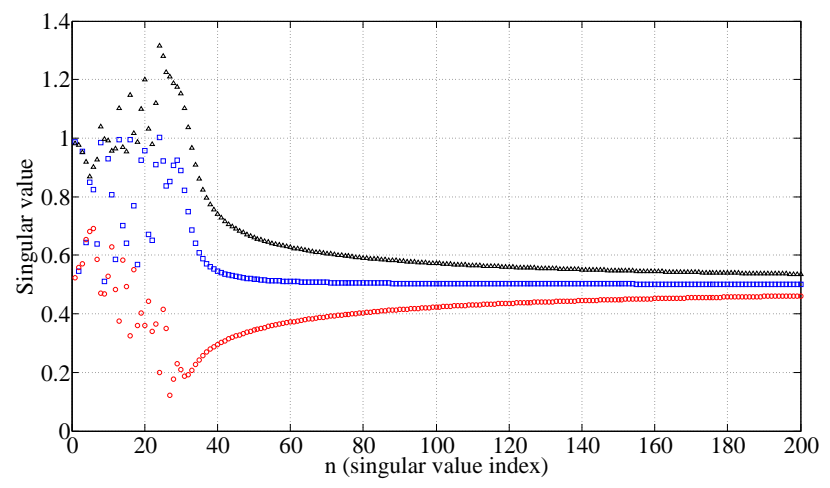

FIGURE 7.2. Singular values of the scaled DPIEv integral equation 7.3 for a spherical scatterer of radius 1 at $k=30$. Three curves are shown, since 7.3 is a vector integral equation with three unknowns (the scalar $\varrho$ and the tangential surface vector a).

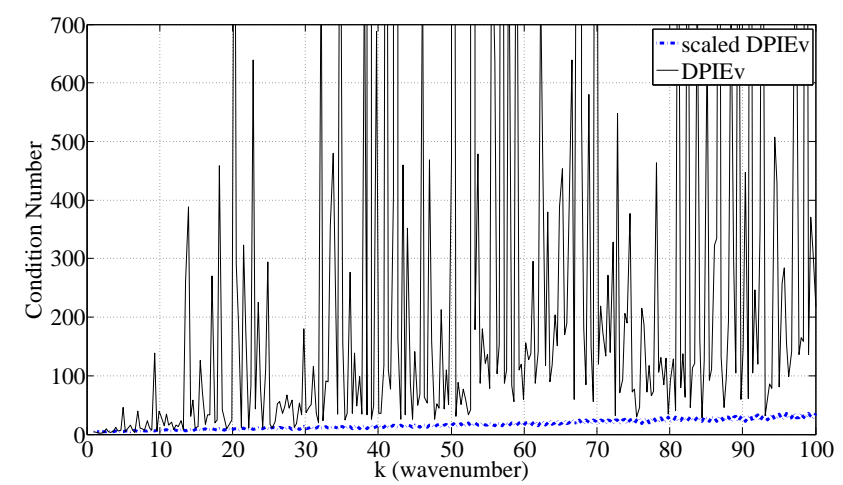

FIGURE 7.3. Condition number of the scaled DPIEv $(7.3)$ and the original DPIEv (3.51) for a spherical scatterer of radius 1 as a function of wavenumber $k$.

\section{Conclusions}

We have presented a new formulation of the problem of electromagnetic scattering from perfect electric conductors. Rather than imposing boundary conditions on the field quantities themselves, we have derived well-posed boundary value problems for the vector and scalar potentials themselves, in the Lorenz gauge. This requires that we describe incoming fields in the same gauge, but that poses no fundamental obstacle. We have explained, in Section 5, how to do this for partial wave expansions, plane waves, and (of course) the potentials induced by known impressed currents and charges. We have also developed integral representations for 


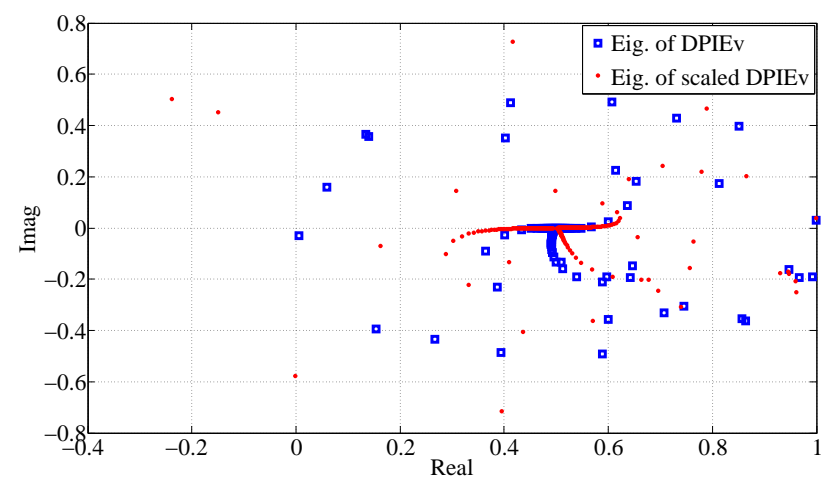

FIGURE 7.4. The spectrum of the original DPIEv and the scaled DPIEv (7.3) for a spherical scatterer of radius 1 at $k=10$.

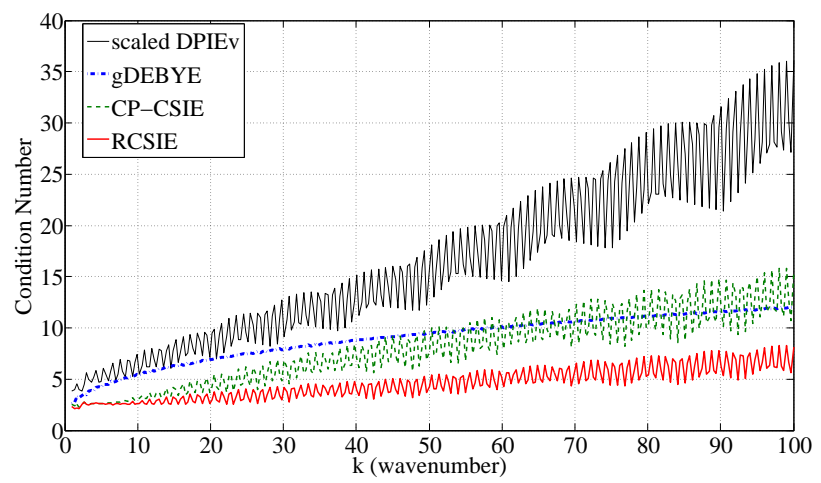

FIGURE 7.5. Comparison of the condition numbers of several resonance-free integral equations: the scaled DPIE, the generalized Debye source equation (gDEBYE) [15], the Calderon preconditioning combined source integral equation (CP-CSIE) [12], and the regularized combined source integral equation (RCSIE) [7].

the vector and scalar potentials that lead to well-conditioned second kind integral equations (the decoupled potential integral equations or DPIE). Most importantly, we have shown that the DPIE is insensitive to the genus of the scatterer. This is one of the few schemes of which we are aware that does not suffer from topological low-frequency breakdown without substantial complications (including the computation of special basis functions that span the space of surface harmonic vector fields [15]). 
Careful analysis of scattering from a unit sphere has shown that the method works well across a range of frequencies, but the DPIE is likely to be of particular utility in the low-frequency regime, especially for structures with complicated, multiply connected geometry. We will report detailed numerical experiments at a later date.

\section{Appendix A Flux Condition}

In the literature, the following strong definition of normal derivative is used:

$$
\frac{\partial \phi}{\partial n}(\boldsymbol{x}):=\lim _{\substack{\delta \rightarrow 0 \\ \delta>0}} \boldsymbol{n}(\boldsymbol{x}) \cdot \nabla \phi(\boldsymbol{x}-\delta \boldsymbol{n}(\boldsymbol{x})), \quad \boldsymbol{x} \in \partial D,
$$

where the limit exists uniformly on $\partial D$ (see [10]). If the function $\phi$ has a normal derivative in that sense, the flux condition 3.12 can be rewritten as

$$
\int_{\partial D_{j}} \frac{\partial \phi}{\partial n} d s=Q_{j}
$$

However, in our formulation of the modified Dirichlet problem we require $\phi$ only to be continuous up to the boundary (which is the natural assumption for a Dirichlet boundary condition). In order to adapt the flux condition to the general case, we make use of the concept of parallel surface (see equation 2.7 in [10]):

$$
\partial D^{\delta}=\{\mathbf{x}=\mathbf{z}+\delta \boldsymbol{n}(\mathbf{z}), \mathbf{z} \in \partial D\}
$$

where $\partial D^{\delta}$ is a well-defined surface of class $C^{1}$ if $\delta$ is restricted to be sufficiently small $0<\delta \leq \delta_{0}$.

Next we prove that the limit 3.12 always exists if $\phi$ is a Helmholtz function continuous up to the boundary.

THEOREM A.1. Let $\phi \in C^{2}\left(\mathbb{R}^{3} \backslash \bar{D}\right) \cap C\left(\mathbb{R}^{3} \backslash D\right)$ be a solution to the Helmholtz or Laplace equation. Then the limit 3.12

$$
\lim _{\delta \rightarrow 0} \int_{\partial D_{j}^{\delta}} \frac{\partial \phi}{\partial n} d s
$$

exists and is finite.

PROOF. Take $\delta_{0}$ sufficiently small (in the sense of parallel surfaces) and consider the quantity $I_{\delta_{0}}$ :

$$
I_{\delta_{0}}=\int_{\partial D_{j}^{\delta_{0}}} \frac{\partial \phi}{\partial n} d s-\int_{D^{\delta_{0} \backslash D}} \Delta \phi d v .
$$

Notice that $I_{\delta_{0}}$ is independent of the particular choice of $\delta_{0}$. (To see this, apply Green's theorem on the region $D^{\delta_{0}} \backslash D^{\delta_{1}}$ and note that $I_{\delta_{0}}-I_{\delta_{1}}=0$ ). Therefore 
the $\operatorname{limit}_{\lim _{\delta \rightarrow 0} I_{\delta}}$ exists and is equal to $I_{\delta_{0}}$. On the other hand, we have that $\phi$ is Helmholtz and bounded up to the boundary, therefore

$$
\begin{aligned}
\lim _{\delta \rightarrow 0}\left|\int_{D^{\delta} \backslash D} \Delta \phi d v\right| & =\lim _{\delta \rightarrow 0}\left|-k^{2} \int_{D^{\delta} \backslash D} \phi d v\right| \\
& \leq \lim _{\delta \rightarrow 0} \operatorname{vol}\left(D^{\delta} \backslash D\right) k^{2}\|\phi\|_{\infty, D^{\delta_{0} \backslash D}}=0 .
\end{aligned}
$$

Therefore we obtain that

$$
\int_{\partial D_{j}^{+}} \frac{\partial \phi}{\partial n} d s=\lim _{\delta \rightarrow 0^{+}} \int_{\partial D_{j}^{\delta}} \frac{\partial \phi}{\partial n} d s=I_{\delta_{0}} .
$$

For the vector case we have a similar situation. The function $\boldsymbol{A}$ might have no normal component continuous up to the boundary, yet the divergence $\nabla \cdot \boldsymbol{A}$ is continuous up to the boundary. This allows us to prove that the limit 3.17 is well-defined (the limit always exists).

Theorem A.2. Let $\boldsymbol{A} \in C^{2}\left(\mathbb{R}^{3} \backslash \bar{D}\right)$ with $\nabla \cdot \boldsymbol{A} \in C\left(\mathbb{R}^{3} \backslash D\right)$; then the flux condition 3.17

$$
\lim _{\delta \rightarrow 0} \int_{\partial D_{j}^{\delta}} \boldsymbol{n} \cdot \boldsymbol{A} d s
$$

exists and is finite.

Again take $\delta_{0}$ sufficiently small. Then

$$
\lim _{\delta \rightarrow 0^{+}} \int_{\partial D_{j}^{\delta}} \boldsymbol{n} \cdot \boldsymbol{A} d s=\int_{\partial D_{j}^{\delta_{0}}} \boldsymbol{n} \cdot \boldsymbol{A} d s-\int_{D^{\delta_{0} \backslash D}} \nabla \cdot \boldsymbol{A} d v .
$$

Similarly, the right-hand side of A.7 is independent of the particular choice of $\delta_{0}$ and

$$
\lim _{\delta \rightarrow 0}\left|\int_{D^{\delta \backslash D}} \nabla \cdot \boldsymbol{A} d v\right| \leq \lim _{\delta \rightarrow 0} \operatorname{vol}\left(D^{\delta} \backslash D\right)\|\nabla \cdot \boldsymbol{A}\|_{\infty, D^{\delta_{0} \backslash D}}=0 .
$$

Next we show some examples of applications of the flux condition that simplify the numerical calculations.

First, consider $\phi_{0}^{\text {scat }}:=D_{0}[\sigma](\mathbf{x})$ for a continuous density $\sigma \in C(\partial D)$ and where the double-layer potential is defined in 3.40 for Laplace. Notice that $D_{0}^{\prime} \sigma$ might not exist for merely continuous densities. Consider the following identity:

$$
\nabla \times \nabla \times S_{0}[\boldsymbol{n} \sigma](\mathbf{x})=\nabla \nabla \cdot S_{0}[\boldsymbol{n} \sigma](\mathbf{x})=-\nabla D_{0}[\sigma](\mathbf{x})
$$


for $\mathbf{x} \in \mathbb{R}^{3} \backslash \bar{D}$. Taking the flux on a parallel surface $\partial D_{j}^{\delta}$ we get

$$
\begin{aligned}
\int_{\partial D_{j}^{\delta}} \boldsymbol{n} \cdot \nabla D_{0} \sigma d s & =\int_{\partial D_{j}^{\delta}}-\boldsymbol{n} \cdot \nabla \times \nabla \times S_{0}(\boldsymbol{n} \sigma) d s \\
& =\int_{\partial D_{j}^{\delta}} \nabla_{s} \cdot \boldsymbol{n} \times \nabla \times S_{0}(\boldsymbol{n} \sigma) d s=0 .
\end{aligned}
$$

Taking the limit, we get

$$
\int_{\partial D_{j}^{+}} D_{0}^{\prime} \sigma d s=\lim _{\delta \rightarrow 0^{+}} \int_{\partial D_{j}^{\delta}} \boldsymbol{n} \cdot \nabla D_{0} \sigma d s=0
$$

Notice that the expression $\boldsymbol{n} \cdot \nabla D_{0} \sigma$ involves off-surface evaluations of the gradient as the density $\sigma$ of the double layer is located at $\partial D$ and the target points are located on $\partial D^{\delta}$.

Another useful example is the double layer for the Helmholtz case, $\phi_{k}^{\text {scat }}:=$ $D_{k}[\sigma](\mathbf{x})$. Proceeding similarly we get

$$
\begin{aligned}
\int_{\partial D_{j}^{+}} D_{k}^{\prime} \sigma d s & =\lim _{\delta \rightarrow 0^{+}} \int_{\partial D_{j}^{\delta}} \boldsymbol{n} \cdot \nabla D_{k} \sigma d s \\
& =\lim _{\delta \rightarrow 0^{+}} \int_{\partial D_{j}^{\delta}} \boldsymbol{n} \cdot \nabla\left(D_{k}-D_{0}\right) \sigma d s=\int_{\partial D_{j}}\left(D_{k}^{\prime}-D_{0}^{\prime}\right) \sigma d s
\end{aligned}
$$

Notice that the operator $D_{k}^{\prime}-D_{0}^{\prime}$ is compact and well- defined for $\sigma \in C(\partial D)$ but $D_{k}^{\prime}$ is only well-defined on $C^{1, \alpha}(\partial D)$.

A third example involves $\boldsymbol{A}^{\text {scat }}=\nabla \times S_{k}[\mathbf{a}](\mathbf{x})$ for continuous tangent density $\mathbf{a} \in C_{t}(\partial D)$. Notice that the normal component $\boldsymbol{n} \cdot \boldsymbol{A}^{\text {scat }}$ is only well-defined up to the boundary for uniformly Hölder-continuous tangent densities $\mathbf{a} \in C_{t}^{0, \alpha}(\partial D)$. Applying the flux condition 3.17 we get

$$
\begin{aligned}
\int_{\partial D_{j}^{+}} \boldsymbol{n} \cdot \nabla \times S_{k}(\mathbf{a}) d s & =\lim _{\delta \rightarrow 0^{+}} \int_{\partial D_{j}^{\delta}} \boldsymbol{n} \cdot \nabla \times S_{k}(\mathbf{a}) d s \\
& =-\lim _{\delta \rightarrow 0^{+}} \int_{\partial D_{j}^{\delta}} \nabla_{s} \cdot \boldsymbol{n} \times S_{k}(\mathbf{a}) d s=0
\end{aligned}
$$




\section{Appendix B Proofs of Existence and Stability Theorems}

Proof of TheOrem 3.13. By construction, the field $\phi^{\text {scat }}(3.43)$ can be extended continuously up to the boundary, $\phi^{\text {scat }} \in C^{2}\left(\mathbb{R}^{3} \backslash \bar{D}\right) \cap C\left(\mathbb{R}^{3} \backslash D\right)$. It satisfies the Helmholtz (or Laplace) equation, the radiation condition, and the flux condition of the modified Dirichlet problem (see observation A.12 of Appendix A.

Consider now a solution $\widetilde{\sigma},\left\{\tilde{V}_{j}\right\}_{j=1}^{N}$ of the homogeneous equation 3.44:

$$
\begin{gathered}
\frac{\tilde{\sigma}}{2}+D_{k} \tilde{\sigma}-i \eta S_{k} \tilde{\sigma}-\sum_{j=1}^{N} \tilde{V}_{j} \chi_{j}=0, \\
\int_{\partial D_{j}}\left(\left(D_{k}^{\prime}-D_{0}^{\prime}\right) \tilde{\sigma}+i \eta \frac{\tilde{\sigma}}{2}-i \eta S_{k}^{\prime} \tilde{\sigma}\right) d s=0 .
\end{gathered}
$$

For this solution, the scalar function and constants

$$
\phi^{\text {scat }}(\boldsymbol{x})=D_{k}[\widetilde{\sigma}](\boldsymbol{x})-i \eta S_{k}[\widetilde{\sigma}](\boldsymbol{x}), \quad\left\{\tilde{V}_{j}\right\}_{j=1}^{N},
$$

satisfy the scalar modified Dirichlet problem with right-hand side $f=0,\left\{Q_{j}=\right.$ $0\}_{j=1}^{N}$. By Theorem 3.9. we have $\phi^{\text {scat }}=0,\left\{\tilde{V}_{j}=0\right\}_{j=1}^{N}$. As $\phi^{\text {scat }}$ is represented by a combination of single and double layers, it is known that $\tilde{\sigma}=0$. This proves uniqueness (see [10,11]). Note that the operators $D_{k}$ and $S_{k}$ defined on $C(\partial D)$ are compact (see [10]). The rest of the operators in (3.44) are finite rank, so that the integral equation is a second-kind equation when acting on the space $C(\partial D) \times \mathbb{C}^{N}$, where $\mathbb{C}^{N}$ is equipped with the usual finite-dimensional topology. By Fredholm theory, for any right-hand side $f,\left\{\widetilde{Q}_{j}\right\}_{j=1}^{N} \in C(\partial D) \times \mathbb{C}^{N}$, there exists a solution $\sigma,\left\{\tilde{V}_{j}\right\}_{j=1}^{N} \in C(\partial D) \times \mathbb{C}^{N}$.

Note also that, as a function of $k$, the operators involved are continuous in the range $k \in\left[0, k_{\max }\right]$ for any fixed $k_{\max }$. This implies that the operators involved in equation (3.44) are not only compact, but collectively compact as well (see [4, 5]). This fact will be used later in the proof of Theorem 3.16 .

Proof of Theorem 3.14. By construction, the field $\boldsymbol{A}^{\text {scat }}$ (3.50) has tangential components that can be extended continuously up to the boundary in the sense of (3.9), and $\boldsymbol{A}^{\text {scat }} \in C^{2}\left(\mathbb{R}^{3} \backslash \bar{D}\right)$, satisfying the Helmholtz (or Laplace) equation, radiation condition, and flux condition. Note that $\nabla \cdot \boldsymbol{A}^{\text {scat }}$ can also be extended continuously up to the boundary.

Consider now a solution $\widetilde{\mathbf{a}}, \widetilde{\varrho},\left\{\widetilde{v}_{j}\right\}_{j=1}^{N}$ of the homogeneous equation (B.3):

$$
\begin{gathered}
\frac{1}{2}\left(\begin{array}{c}
\widetilde{\mathbf{a}} \\
\widetilde{\varrho}
\end{array}\right)+\overline{\bar{L}}\left(\begin{array}{c}
\widetilde{\mathbf{a}} \\
\tilde{\varrho}
\end{array}\right)+i \eta \overline{\bar{R}} \overline{\bar{S}}_{i k}\left(\begin{array}{c}
\widetilde{\mathbf{a}} \\
\tilde{\varrho}
\end{array}\right)-\left(\begin{array}{c}
0 \\
\sum_{j=1}^{N} v_{j} \chi_{j}
\end{array}\right)=\left(\begin{array}{l}
\mathbf{0} \\
0
\end{array}\right), \\
\int_{\partial D_{j}}\left(-\boldsymbol{n} \cdot S_{k}(\boldsymbol{n} \widetilde{\varrho})+i \eta\left(\boldsymbol{n} \cdot S_{k}\left(\mathbf{n} \times S_{i k} \widetilde{\mathbf{a}}\right)-\frac{S_{i k} \widetilde{\varrho}}{2}+S_{k}^{\prime}\left(S_{i k} \widetilde{\varrho}\right)\right)\right) d s=0 .
\end{gathered}
$$


For this solution, the vector field and constants

$$
\begin{aligned}
\boldsymbol{A}^{\text {scat }}= & \nabla \times S_{k}[\widetilde{\mathbf{a}}](\boldsymbol{x})-S_{k}[\boldsymbol{n} \widetilde{\varrho}](\boldsymbol{x}) \\
& +i \eta\left(S_{k}\left[\boldsymbol{n} \times S_{i k} \widetilde{\mathbf{a}}\right](\boldsymbol{x})+\nabla S_{k}\left[S_{i k} \widetilde{\varrho}\right](\boldsymbol{x})\right), \quad\left\{\tilde{v}_{j}\right\}_{j=1}^{N},
\end{aligned}
$$

satisfy the vector modified Dirichlet problem, with right-hand side $\mathbf{f}=0, h=$ $0,\left\{q_{j}=0\right\}_{j=1}^{N}$. By Theorem 3.10, we have $\boldsymbol{A}^{\text {scat }}(\mathbf{x})=0$ for $\mathbf{x} \in \mathbb{R}^{3} \backslash \bar{D}$ and $\left\{\tilde{V}_{j}=0\right\}_{j=1}^{N}$. Using the jump conditions and the vector version of Green's theorem as in theorem 4.42 of [10] or theorem 6.21 of [11], we obtain

(B.5) $i \eta \int_{\partial D} \overline{\widetilde{\mathbf{a}}} \cdot S_{i k} \tilde{\mathbf{a}}+\overline{\widetilde{\varrho}} S_{i k} \tilde{\varrho} d s=\int_{D}\left|\nabla \times \boldsymbol{A}^{\text {scat }}\right|^{2}+\left|\nabla \cdot \boldsymbol{A}^{\text {scat }}\right|^{2}-k^{2}\left|\boldsymbol{A}^{\text {scat }}\right| d v$.

Note that we can apply the jump conditions for $\boldsymbol{n} \times \nabla \times S_{k}$ (a) with only continuous densities in the sense of theorem 2.26 of [10]. Taking imaginary parts, we obtain

$$
\eta \int_{\partial D} \overline{\widetilde{\mathbf{a}}} \cdot S_{i k} \tilde{\mathbf{a}}+\overline{\widetilde{\varrho}} S_{i k} \widetilde{\varrho} d s=0 .
$$

Due to the coercivity of the operators $S_{i k} \mathbf{a}$ and $S_{i k} \varrho$, we obtain $\widetilde{\mathbf{a}}=0, \widetilde{\varrho}=0$, and uniqueness follows.

Note now that the operator $\overline{\bar{L}}: C_{t}(\partial D) \times C(\partial D) \rightarrow C_{t}(\partial D) \times C(\partial D)$ is compact (see [10]). The operator $\overline{\bar{R}}$ is continuous and bounded on $C_{t}^{0, \alpha}(\partial D) \times C^{0, \alpha}(\partial D)$, and the operator $\overline{\bar{S}}_{i k}$ is continuous as a map from $C_{t}(\partial D) \times C(\partial D)$ to $C_{t}^{0, \alpha}(\partial D) \times$ $C^{0, \alpha}(\partial D)$. The resulting operator $\overline{\bar{L}}+i \eta \overline{\bar{R}} \overline{\bar{S}}_{i k}$ is compact on $C_{t}(\partial D) \times C(\partial D)$.

The remaining operators in equation (B.3) are finite rank, so that equation (B.3) is second kind when acting on the space $C_{t}(\partial D) \times C(\partial D) \times \mathbb{C}^{N}$, where $\mathbb{C}^{N}$ is equipped with the usual finite-dimensional topology. By Fredholm theory, for any right-hand side $\mathbf{f}, h,\left\{\tilde{q}_{j}\right\}_{j=1}^{N} \in C_{t}(\partial D) \times C(\partial D) \times \mathbb{C}^{N}$, there exists a solution $\mathbf{a}, \varrho,\left\{\tilde{v}_{j}\right\}_{j=1}^{N} \in C_{t}(\partial D) \times C(\partial D) \times \mathbb{C}^{N}$.

Note also that, as a function of $k$, the operators involved are continuous in the range $k \in\left[0, k_{\max }\right]$ for any fixed $k_{\max }$. This implies that the operators involved in equation (B.3) are not only compact, but collectively compact [4]. This fact will be used to prove Theorem 3.17 .

Proof OF THEOREM 3.16. The operator that appears in equation 3.44 is second kind as a map from $C(\partial D) \times \mathbb{C}^{N}$ to $C(\partial D) \times \mathbb{C}^{N}$ and the equation is uniquely solvable. Thus, the inverse operator exists and is bounded from $C(\partial D) \times \mathbb{C}^{N}$ to $C(\partial D) \times \mathbb{C}^{N}$. Due to the collective compactness of the operators, the continuity of the inverse is uniform in $\left[0, k_{\max }\right]$. This, together with the regularity properties of 3.40 (see [10]), implies the estimate 3.52 .

By the same argument, the operator 3.44 is also second kind from $C^{0, \alpha}(\partial D) \times$ $\mathbb{C}^{N}$ to $C^{0, \alpha}(\partial D) \times \mathbb{C}^{N}$, so that the inverse is uniformly bounded from $C^{0, \alpha}(\partial D) \times$ 
$\mathbb{C}^{N}$ to $C^{0, \alpha}(\partial D) \times \mathbb{C}^{N}$ in the interval $\left[0, k_{\max }\right]$. Using again the regularity properties of the operators 3.40 , we obtain the estimate 3.53

Likewise, the operator (3.44) is compact from $C^{1, \alpha}(\partial D) \times \mathbb{C}^{N}$ to $C^{1, \alpha}(\partial D) \times$ $\mathbb{C}^{N}$ (see [11]). This implies 3.54

Proof of THEOREM 3.17. The operator that appears in equation (3.51) is second kind as a map from $C_{t}(\partial D) \times C(\partial D) \times \mathbb{C}^{N}$ to $C_{t}(\partial D) \times C(\partial D) \times \mathbb{C}^{N}$ and uniquely solvable. Thus, the inverse operator exists and is bounded from $C_{t}(\partial D) \times C(\partial D) \times \mathbb{C}^{N}$ to $C_{t}(\partial D) \times C(\partial D) \times \mathbb{C}^{N}$. Due to the collective compactness of the operators, the continuity of the inverse is uniform in $\left[0, k_{\max }\right]$. This, together with the regularity properties of the operators 3.46, 3.48, and 3.49 (see [10]) implies the estimates 3.55 and 3.56

A similar argument taking into account that equation (3.51) is also second kind from $C_{t}^{0, \alpha}(\partial D) \times C^{0, \alpha}(\partial D) \times \mathbb{C}^{N}$ to $C_{t}^{0, \alpha}(\partial D) \times C^{0, \alpha}(\partial D) \times \mathbb{C}^{N}$ implies the estimations 3.57 and 3.58

Notice the difference between 3.55 and 3.57 . We need to use the set $\mathfrak{M} \subset \mathbb{R}^{3} \backslash \bar{D}$ in 3.55, as the vector field $F:=\nabla \times S_{k}(\mathbf{a}), \mathbf{a} \in C_{t}(\partial D)$, might not have a well-defined normal component for merely continuous density $\mathbf{a} \in C_{t}(\partial D)$. In contrast, for uniformly Hölder-continuous tangent densities $\mathbf{a} \in C^{0, \alpha}(\partial D)$, the vector field $F$ can be extended in a uniformly Hölder-continuous way up to the boundary $F \in C^{0, \alpha}\left(\mathbb{R}^{3} \backslash D\right)$.

Again taking into account that equation (3.51) is second kind from

$$
C_{t}^{0, \alpha}(\operatorname{div}, \partial D) \times C^{0, \alpha}(\partial D) \times \mathbb{C}^{N} \text { to } C_{t}^{0, \alpha}(\operatorname{div}, \partial D) \times C^{0, \alpha}(\partial D) \times \mathbb{C}^{N}
$$

and uniquely solvable, the inverse operator exists and is bounded from

$$
C_{t}^{0, \alpha}(\operatorname{div}, \partial D) \times C^{0, \alpha}(\partial D) \times \mathbb{C}^{N} \quad \text { to } \quad C_{t}^{0, \operatorname{div}}(\operatorname{div}, \partial D) \times C^{0, \alpha}(\partial D) \times \mathbb{C}^{N} .
$$

Notice that the operator $\overline{\bar{L}}$ is only continuous with the given norm $C_{t}^{0, \alpha}(\operatorname{div}, \partial D) \times$ $C^{0, \alpha}(\partial D)$. In particular, $L_{12}(\rho)=-\boldsymbol{n} \times S_{k}(\boldsymbol{n} \rho)$ is only continuous, not compact (note that $\nabla_{s} \cdot L_{12}(\rho)=\nabla_{s} \cdot\left(-\boldsymbol{n} \times S_{k}(\boldsymbol{n} \rho)\right)=\boldsymbol{n} \cdot \nabla \times S_{k}(\boldsymbol{n} \rho)$; see [11]). Yet the rest of the operators are compact and we can write the operator $\frac{1}{2}+\bar{L}$ in (3.51) in the form

$$
\frac{1}{2}\left(\begin{array}{c}
\mathbf{a} \\
\rho
\end{array}\right)+\overline{\bar{L}}\left(\begin{array}{c}
\mathbf{a} \\
\rho
\end{array}\right)=\left(\begin{array}{cc}
\frac{1}{2} & L_{12} \\
0 & \frac{1}{2}
\end{array}\right)\left(\begin{array}{l}
\mathbf{a} \\
\rho
\end{array}\right)+\left(\begin{array}{cc}
L_{11} & 0 \\
L_{21} & L_{22}
\end{array}\right)\left(\begin{array}{l}
\mathbf{a} \\
\rho
\end{array}\right)
$$

where the first term is invertible with inverse

$$
\left(\begin{array}{cc}
\frac{1}{2} & L_{12} \\
0 & \frac{1}{2}
\end{array}\right)^{-1}=\left(\begin{array}{cc}
2 & -4 L_{12} \\
0 & 2
\end{array}\right)
$$

and the rest is compact. Equations 3.59, 3.60, and 3.61 follow. 


\section{Appendix C Partial Wave Expansions}

An important representation of the electromagnetic field is that based on separation of variables in spherical coordinates. As shown independently by Lorenz, Debye, and Mie [15,26], the fields induced by sources in the interior of a sphere can always be expressed in the exterior of the sphere according to the representation

$$
\begin{aligned}
\boldsymbol{E}^{\mathrm{far}} & =\sum_{m, n}\left[a_{m n} \nabla \times \nabla \times\left(\boldsymbol{x} h_{n}(k|\boldsymbol{x}|) Y_{n}^{m}\right)+i \omega \mu b_{m n} \nabla \times\left(\boldsymbol{x} f_{n}(k|\boldsymbol{x}|) Y_{n}^{m}\right)\right], \\
\boldsymbol{H}^{\mathrm{far}} & =\sum_{m, n}\left[b_{m n} \nabla \times \nabla \times\left(\boldsymbol{x} h_{n}(k|\boldsymbol{x}|) Y_{n}^{m}\right)-i \omega \epsilon a_{m n} \nabla \times\left(\boldsymbol{x} f_{n}(k|\boldsymbol{x}|) Y_{n}^{m}\right)\right],
\end{aligned}
$$

where $h_{n}$ is the spherical Hankel function of the first kind. For sources in the exterior of the sphere, we have

$$
\begin{aligned}
\boldsymbol{E}^{\mathrm{loc}} & =\sum_{m, n}\left[a_{m n} \nabla \times \nabla \times\left(\boldsymbol{x} j_{n}(k|\boldsymbol{x}|) Y_{n}^{m}\right)+i \omega \mu b_{m n} \nabla \times\left(\boldsymbol{x} f_{n}(k|\boldsymbol{x}|) Y_{n}^{m}\right)\right], \\
\boldsymbol{H}^{\mathrm{loc}} & =\sum_{m, n}\left[b_{m n} \nabla \times \nabla \times\left(\boldsymbol{x} j_{n}(k|\boldsymbol{x}|) Y_{n}^{m}\right)-i \omega \epsilon a_{m n} \nabla \times\left(\boldsymbol{x} f_{n}(k|\boldsymbol{x}|) Y_{n}^{m}\right)\right],
\end{aligned}
$$

where $j_{n}$ is the spherical Bessel function [1].

In order to obtain a finite static limit, we renormalize and define the modified spherical Hankel/Bessel function by

$$
\tilde{f}_{n}(k, r):=\left\{\begin{array}{l}
\tilde{h}_{n}(k, r)=h_{n}(k r) \frac{k^{n+1}}{-i(2 n-1)(2 n-3) \cdots 3 \cdot 1}, \\
\tilde{j}_{n}(k, r)=j_{n}(k r) \frac{(2 n+1)(2 n-1) \cdots 3 \cdot 1}{k^{n+1}} .
\end{array}\right.
$$

It is easy to check that

$$
\lim _{k \rightarrow 0} \tilde{f}_{n}(k, r)=\left\{\begin{array}{l}
\lim _{k \rightarrow 0} \tilde{h}_{n}(k, r)=\frac{1}{r^{n+1}}, \\
\lim _{k \rightarrow 0} \tilde{j}_{n}(k, r)=r^{n}
\end{array}\right.
$$

With a slight abuse of notation we will refer to both $\boldsymbol{E}^{\text {far }}$ and $\boldsymbol{E}^{\text {loc }}$ as $\boldsymbol{E}^{\text {inc }}$, and to both $\boldsymbol{H}^{\text {far }}$ and $\boldsymbol{H}^{\text {loc }}$ as $\boldsymbol{H}^{\text {inc }}$. When the distinction is important, we will specify the use of $\tilde{h}_{n}(k, r)$ or $\widetilde{j}_{n}(k, r)$ as the radial function of interest.

Normalizing the coefficients $a_{m n}, b_{m n}$ by the inverse of the scaling factor in (C.3), we write

$$
\begin{aligned}
\boldsymbol{E}^{\mathrm{inc}} & =\sum_{m, n}\left[\widetilde{a}_{m n} \nabla \times \nabla \times\left(\boldsymbol{x} \tilde{f}_{n}(k,|\boldsymbol{x}|) Y_{n}^{m}\right)+i \omega \mu \widetilde{b}_{m n} \nabla \times\left(\boldsymbol{x} \tilde{f}_{n}(k,|\boldsymbol{x}|) Y_{n}^{m}\right)\right], \\
\boldsymbol{H}^{\mathrm{inc}} & =\sum_{m, n}\left[\widetilde{b}_{m n} \nabla \times \nabla \times\left(\boldsymbol{x} \widetilde{f}_{n}(k,|\boldsymbol{x}|) Y_{n}^{m}\right)-i \omega \epsilon \widetilde{a}_{m n} \nabla \times\left(\boldsymbol{x} \widetilde{f}_{n}(k,|\boldsymbol{x}|) Y_{n}^{m}\right)\right] .
\end{aligned}
$$

The fields of a magnetic multipole of degree $n$ and order $m$ are defined to be

$$
\begin{aligned}
\dot{\boldsymbol{E}}_{n m}^{\text {inc }} & =i \omega \mu \nabla \times\left(\boldsymbol{x} \tilde{f}_{n}(k,|\boldsymbol{x}|) Y_{n}^{m}\right), \\
\dot{\boldsymbol{H}}_{n m}^{\text {inc }} & =\nabla \times \nabla \times\left(\boldsymbol{x} \tilde{f}_{n}(k,|\boldsymbol{x}|) Y_{n}^{m}\right) .
\end{aligned}
$$


The corresponding vector and scalar potentials can be defined by

$$
\dot{\boldsymbol{A}}_{n m}^{\text {inc }}=\mu \nabla \times\left(\boldsymbol{x} \tilde{f}_{n}(k,|\boldsymbol{x}|) Y_{n}^{m}\right), \quad \dot{\phi}_{n m}^{\text {inc }}=0 .
$$

They clearly satisfy

$$
\begin{gathered}
\Delta \dot{\phi}_{n m}^{\mathrm{inc}}+k^{2} \dot{\phi}_{n m}^{\mathrm{inc}}=0, \quad \Delta \dot{\boldsymbol{A}}_{n m}^{\mathrm{inc}}+k^{2} \dot{\boldsymbol{A}}_{n m}^{\mathrm{inc}}=0, \\
\nabla \cdot \dot{\boldsymbol{A}}_{n m}^{\mathrm{inc}}=i \omega \mu \epsilon \dot{\phi}_{n m}^{\mathrm{inc}} .
\end{gathered}
$$

Moreover, $\dot{\boldsymbol{A}}_{m n}^{\text {inc }}$ and $\dot{\phi}_{m n}^{\text {inc }}$ are bounded. The fields of an electric multipole of degree $n$ and order $m$ are defined to be

$$
\begin{aligned}
& \ddot{\boldsymbol{E}}_{n m}^{\mathrm{inc}}=\nabla \times \nabla \times\left(\boldsymbol{x} \tilde{f}_{n}(k,|\boldsymbol{x}|) Y_{n}^{m}\right), \\
& \ddot{\boldsymbol{H}}_{n m}^{\mathrm{inc}}=-i \omega \epsilon \nabla \times\left(\boldsymbol{x} \tilde{f}_{n}(k,|\boldsymbol{x}|) Y_{n}^{m}\right) .
\end{aligned}
$$

In this case, however, it is easy to verify that the function that serves as the obvious vector potential, namely $\boldsymbol{x} \widetilde{f}_{n}(k,|\boldsymbol{x}|) Y_{n}^{m}$, is not in the Lorenz gauge. To find a suitable replacement, we compute

$$
\begin{aligned}
\nabla \times \nabla \times\left(\boldsymbol{x} \tilde{f}_{n}(k,|\boldsymbol{x}|) Y_{n}^{m}\right)= & k^{2} \boldsymbol{x} \tilde{f}_{n}(k,|\boldsymbol{x}|) Y_{n}^{m} \\
& +\nabla \frac{\partial}{\partial r}\left(r \tilde{f}_{n}(k, r) Y_{n}^{m}\right)_{r=|\boldsymbol{x}|} .
\end{aligned}
$$

Note that

$$
\frac{\partial}{\partial r}\left(r \tilde{f}_{n}(k, r) Y_{n}^{m}\right)=\tilde{f}_{n}(k, r) Y_{n}^{m}+r \frac{\partial}{\partial r} \tilde{f}_{n}(k, r) Y_{n}^{m} .
$$

The first term $\tilde{f}_{n}(k, r) Y_{n}^{m}$ is a Helmholtz potential. Making use of the following identity for spherical Hankel and Bessel functions [1]

(C.12) $\frac{n+1}{z} h_{n}(z)+h_{n}^{\prime}(z)=h_{n-1}(z), \quad \frac{n}{z} j_{n}(z)-j_{n}^{\prime}(z)=j_{n+1}(z)$,

we have

$$
r \frac{\partial}{\partial r} \tilde{h}_{n}(k, r)=\frac{r k^{2}}{2 n-1} \tilde{h}_{n-1}(k, r)-(n+1) \tilde{h}_{n}(k, r)
$$

and

$$
r \frac{\partial}{\partial r} \tilde{j}_{n}(k, r)=-\frac{r k^{2}}{2 n+3} \tilde{j}_{n+1}(k, r)+n \tilde{j}_{n}(k, r) .
$$

Multiplying by $Y_{n}^{m}$ leads to

$$
\begin{aligned}
& r \frac{\partial}{\partial r} \tilde{h}_{n}(k, r) Y_{n}^{m}=\frac{r k^{2}}{2 n-1} \tilde{h}_{n-1}(k, r) Y_{n}^{m}-(n+1) \tilde{h}_{n}(k, r) Y_{n}^{m}, \\
& r \frac{\partial}{\partial r} \widetilde{j}_{n}(k, r) Y_{n}^{m}=-\frac{r k^{2}}{2 n+3} \widetilde{j}_{n+1}(k, r) Y_{n}^{m}+n \tilde{j}_{n}(k, r) Y_{n}^{m} .
\end{aligned}
$$


The first term on the right-hand side is of the order $O(k)$, while the second term is a Helmholtz potential and of magnitude $O(1)$. Using (C.15) and (C.11) in (C.10), we obtain

$$
\begin{aligned}
\nabla \times & \nabla \times\left(\boldsymbol{x} \tilde{h}_{n}(k,|\boldsymbol{x}|) Y_{n}^{m}\right) \\
= & k^{2} \boldsymbol{x} \tilde{h}_{n}(k,|\boldsymbol{x}|) Y_{n}^{m}+\frac{k^{2}}{2 n-1} \nabla\left(r \tilde{h}_{n-1}(k, r) Y_{n}^{m}\right)_{r=|\boldsymbol{x}|} \\
& -\nabla\left(n \tilde{h}_{n}(k, r) Y_{n}^{m}\right)_{r=|\boldsymbol{x}|}
\end{aligned}
$$

for the outgoing waves, and

$$
\begin{aligned}
\nabla \times & \nabla \times\left(\boldsymbol{x} \tilde{j}_{n}(k,|\boldsymbol{x}|) Y_{n}^{m}\right) \\
= & k^{2} \boldsymbol{x} \tilde{j}_{n}(k,|\boldsymbol{x}|) Y_{n}^{m}-\frac{k^{2}}{2 n+3} \nabla\left(r \tilde{j}_{n+1}(k, r) Y_{n}^{m}\right)_{r=|\boldsymbol{x}|} \\
& +\nabla\left((n+1) \tilde{j}_{n}(k, r) Y_{n}^{m}\right)_{r=|\boldsymbol{x}|}
\end{aligned}
$$

for the incoming waves. Note that the last term on the right-hand side of (C.17) and the left-hand side of (C.17) both satisfy the vector Helmholtz equation. Thus, the first two terms on the right-hand side of (C.17) must together satisfy the vector Helmholtz equation as well. Dividing those two terms by $k^{2}$ and multiplying by $-i \omega \mu \epsilon$, we define the corresponding vector and scalar potentials by

$$
\begin{aligned}
\ddot{\boldsymbol{A}}_{n m}^{\text {inc }} & =-i \omega \mu \epsilon \boldsymbol{x} \tilde{h}_{n}(k,|\boldsymbol{x}|) Y_{n}^{m}+\frac{-i \omega \mu \epsilon}{2 n-1} \nabla\left(r \tilde{h}_{n-1}(k, r) Y_{n}^{m}\right)_{r=|\boldsymbol{x}|}, \\
\ddot{\phi}_{n m}^{\text {inc }} & =n \tilde{h}_{n}(k,|\boldsymbol{x}|) Y_{n}^{m},
\end{aligned}
$$

for the outgoing waves and

$$
\begin{aligned}
\ddot{\boldsymbol{A}}_{n m}^{\mathrm{inc}} & =-i \omega \mu \epsilon \boldsymbol{x}{\tilde{j_{n}}}_{n}(k,|\boldsymbol{x}|) Y_{n}^{m}+\frac{i \omega \mu \epsilon}{2 n+3} \nabla\left(r{\tilde{j_{n}-1}}(k, r) Y_{n}^{m}\right)_{r=|\boldsymbol{x}|}, \\
\ddot{\phi}_{n m}^{\text {inc }} & =-(n+1) \tilde{j}_{n}(k,|\boldsymbol{x}|) Y_{n}^{m},
\end{aligned}
$$

for the incoming waves. It is easy to verify that

$$
\begin{gathered}
\Delta \ddot{\phi}_{n m}^{\mathrm{inc}}+k^{2} \ddot{\phi}_{n m}^{\mathrm{inc}}=0, \quad \Delta \ddot{\boldsymbol{A}}_{n m}^{\mathrm{inc}}+k^{2} \ddot{\boldsymbol{A}}_{n m}^{\mathrm{inc}}=0, \\
\nabla \cdot \ddot{\boldsymbol{A}}_{n m}^{\mathrm{inc}}=i \omega \mu \epsilon \ddot{\phi}_{n m}^{\mathrm{inc}} .
\end{gathered}
$$

The last equation, which enforces the Lorenz gauge, is obtained by taking the divergence of (C.17). Clearly, both potentials $\ddot{A}_{m n}^{\text {inc }}$ and $\ddot{\phi}_{m n}^{\text {inc }}$ are of the order $O(1)$.

Acknowledgment. This work was supported in part by the Applied Mathematical Sciences Program of the U.S. Department of Energy under Contract DEFGO288ER25053 (L.G.), by the Office of the Assistant Secretary of Defense for Research and Engineering and AFOSR under NSSEFF Program Award FA955010-1-0180 (L.G. and Z. G.), and in part by the Spanish Ministry of Science and Innovation (Ministerio de Ciencia e Innovacion) under projects CSD2008-00068 
and TEC2010-20841-C04-01. The authors thank A. Klöckner and M. O'Neil for many useful discussions.

\section{Bibliography}

[1] Abramowitz, M.; Stegun, I. A. Handbook of mathematical functions: with formulas, graphs, and mathematical tables. Dover, New York, 1964.

[2] Aharonov, Y.; Bohm, D. Significance of electromagnetic potentials in the quantum theory. Phys. Rev. (2) 115 (1959), 485-491.

[3] Andriulli, F. P.; Cools, K.; Bogaert, I.; Michielssen, E. On a well-conditioned electric field integral operator for multiply connected geometries. IEEE Trans. Antennas and Propagation 61 (2013), no. 4, part 2, 2077-2087. doi:10.1109/TAP.2012.2234072

[4] Anselone, P. M. Uniform approximation theory for integral equations with discontinuous kernels. SIAM J. Numer. Anal. 4 (1967), 245-253. doi:10.1137/0704023

[5] Atkinson, K. E. The numerical solution of integral equations of the second kind. Cambridge Monographs on Applied and Computational Mathematics, 4. Cambridge University Press, Cambridge, 1997. doi:10.1017/CBO9780511626340

[6] Bendali, A.; Collino, F.; Fares, M.; Steif, B. Extension to nonconforming meshes of the combined current and charge integral equation. IEEE Trans. Antennas and Propagation 60 (2012), no. 10, 4732-4744. doi:10.1109/TAP.2012.2207316

[7] Bruno, O.; Elling, T.; Paffenroth, R.; Turc, C. Electromagnetic integral equations requiring small numbers of Krylov-subspace iterations. J. Comput. Phys. 228 (2009), no. 17, 6169-6183. doi:10.1016/j.jcp.2009.05.020

[8] Calderón, A. P. The multipole expansion of radiation fields. J. Rational Mech. Anal. 3 (1954), 523-537.

[9] Chew, W. C. Waves and fields in inhomogeneous media. IEEE Press, New York, 1995.

[10] Colton, D. L.; Kress, R. Integral equation methods in scattering theory. Wiley, New York, 1983.

[11] Colton, D.; Kress, R. Inverse acoustic and electromagnetic scattering theory. Third edition. Applied Mathematical Sciences, 93. Springer, New York, 2013. doi:10.1007/978-1-4614-49423

[12] Contopanagos, H.; Dembart, B.; Epton, M.; Ottusch, J. J.; Rokhlin, V.; Visher, J. L.; Wandzura, S. M. Well-conditioned boundary integral equations for three-dimensional electromagnetic scattering. IEEE Trans. Antennas and Propagation 50 (2002), no. 12, 1824-1830. doi:10.1109/TAP.2002.803956

[13] Cools, K.; Andriulli, F. P.; Olyslager, F.; Michielssen, E. Nullspaces of MFIE and Calderón preconditioned EFIE operators applied to toroidal surfaces. IEEE Trans. Antennas and Propagation 57 (2009), no. 10, part 2, 3205-3215. doi:10.1109/TAP.2009.2028669

[14] Epstein, C. L.; Gimbutas, Z.; Greengard, L.; Klöckner, A.; O’Neil, M. A consistency condition for the vector potential in multiply-connected domains. IEEE Transactions on Magnetics 49 (2013), no. 3, 1072-1076. doi:10.1109/TMAG.2012.2223480

[15] Epstein, C. L.; Greengard, L. Debye sources and the numerical solution of the time harmonic Maxwell equations. Comm. Pure Appl. Math. 63 (2010), no. 4, 413-463. doi:10.1002/cpa.20313

[16] Hähner, P. Eindeutigkeits- und Regularitätssätze für Randwertprobleme bei der skalaren und vektoriellen Helmholtz-Gleichung. Doctoral dissertation, Göttingen, 1990.

[17] Jackson, J. D. Classical electrodynamics. Second edition. Wiley, New York-London-Sydney, 1975.

[18] Jin, J.-M. Theory and computation of electromagnetic fields. Wiley, Hoboken, N.J.; IEEE Press, New York, 2010. doi:10.1002/9780470874257 
[19] Kress, R. On the limiting behaviour of solutions to boundary integral equations associated with time harmonic wave equations for small frequencies. Math. Methods Appl. Sci. 1 (1979), no. 1, 89-100. doi:10.1002/mma.1670010108

[20] Kress, R. A singular perturbation problem for linear operators with an application to the limiting behaviour of stationary electromagnetic wave fields for small frequencies. Methods and techniques of mathematical physics (Oberwolfach, 1980), 5-30. Methoden und Verfahren der mathematischen Physik, 21. Lang, Frankfurt, 1981.

[21] Kress, R. Minimizing the condition number of boundary integral operators in acoustic and electromagnetic scattering. Quart. J. Mech. Appl. Math. 38 (1985), no. 2, 323-341. doi:10.1093/qjmam/38.2.323

[22] Lee, J.-F.; Lee, R.; Burkholder, R. J. Loop star basis functions and a robust preconditioner for EFIE scattering problems. IEEE Trans. Antennas and Propagation 51 (2003), no. 8, 1855-1863. doi:10.1109/TAP.2003.814736

[23] Maue, A. W. On the formulation of a general scattering problem by means of an integral equation. Z. Phys 126 (1949), no. 7, 601-618.

[24] Müller, C. Foundations of the mathematical theory of electromagnetic waves. Die Grundlehren der mathematischen Wissenschaften, 155. Springer, New York-Heidelberg, 1969.

[25] Nédélec, J.-C. Acoustic and electromagnetic equations: Integral representations for harmonic problems. Springer, New York, 2001.

[26] Papas, C. H. Theory of electromagnetic wave propagation. Dover, New York, 1988.

[27] Taskinen, M.; Vänskä, S. Current and charge integral equation formulations and Picard's extended Maxwell system. IEEE Trans. Antennas and Propagation 55 (2007), no. 12, 3495-3503. doi:10.1109/TAP.2007.910363

[28] Taskinen, M.; Ylä-Oijala, P. Current and charge integral equation formulation. IEEE Trans. Antennas and Propagation 54 (2006), no. 1, 58-67. doi:10.1109/TAP.2005.861580

[29] Vecchi, G. Loop-star decomposition of basis functions in the discretization of the EFIE. IEEE Trans. Antennas and Propagation 47 (1999), no. 2, 339-346. doi:10.1109/8.761074

[30] Vico, F.; Gimbutas, Z.; Greengard, L.; Ferrando-Bataller, M. Overcoming low-frequency breakdown of the magnetic field integral equation. IEEE Trans. Antennas and Propagation 61 (2013), no. 3, 1285-1290. doi:10.1109/TAP.2012.2230232

[31] Vico, F.; Greengard, L.; Gimbutas, Z. Boundary integral equation analysis on the sphere. Numer. Math. 128 (2014), no. 3, 463-487. doi:10.1007/s00211-014-0619-z

[32] Werner, P. On an integral equation in electromagnetic diffraction theory. J. Math. Anal. Appl. 14 (1966), 445-462. doi:10.1016/0022-247X(66)90005-9

[33] Wilton, D. R.; Glisson, A. W. On improving the electric field integral equation at low frequencies. Proc. URSI Radio Sci. Meet. Digest 24 (1981).

[34] Wu, W.-L.; Glisson, A. W.; Kajfez, D. A study of two numerical solution procedures for the electric field integral equation at low frequency. Applied Computational Electromagnetics Society Journal 10 (1995), no. 3, 69-80.

[35] Yla-Oijala, P.; Taskinen, M.; Jarvenpaa, S. Advanced surface integral equation methods in computational electromagnetics. International Conference on Electromagnetics in Advanced Applications (Torina, 2009), 369-372. IEEE, 2009. doi:10.1109/ICEAA.2009.5297415

[36] Zhang, Y.; Cui, T. J.; Chew, W. C.; Zhao, J.-S. Magnetic field integral equation at very low frequencies. IEEE Trans. Antennas and Propagation 51 (2003), no. 8, 1864-1871. doi:10.1109/TAP.2003.814753

[37] Zhao, J.-S.; Chew, W. C. Integral equation solution of Maxwell's equations from zero frequency to microwave frequencies. IEEE Trans. Antennas and Propagation 48 (2000), no. 10, 16351645. doi: $10.1109 / 8.899680$ 
FELIPE VICO

Instituto de Telecomunicaciones y Aplicaciones Multimedia (ITEAM)

Universidad Politècnica de València 46022 València

SPAIN

E-mail: felipe.vico@gmail.com

LESLIE GREENGARD

Simons Center for Data Analysis, Simons Foundation

Courant Institute

251 Mercer St.

New York, NY 10012-1110

E-mail: greengardecims.nyu.edu USA

Received April 2014.

Revised April 2015.
Miguel FERRANDO

Instituto de Telecomunicaciones y Aplicaciones Multimedia (ITEAM) Universidad Politècnica de València 46022 València

SPAIN

E-mail: mferrandedcom.upv.es

ZYDRUNAS GIMBUTAS

Information Technology Laboratory

National Institute of Standards and Technology 325 Broadway, Mail Stop 891.01 Boulder, CO 80305-3328

USA

E-mail: zydrunas.gimbutase

nist.gov 